https://doi.org/10.30910/turkjans.556594

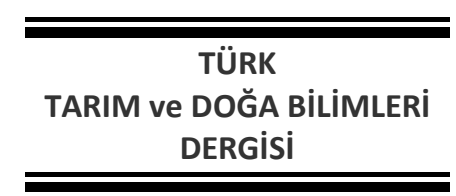

TÜRK

DERGISI

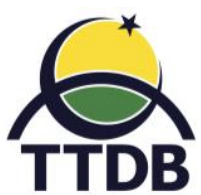

www.dergipark.gov.tr/turkjans

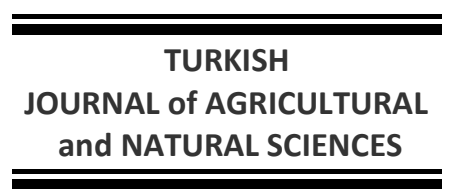

Araştırma Makalesi

\title{
Sulama Kuyularında Çakıl Zonu Genişliklerinin Kritik Dalma Derinliğine ve Vorteks Oluşumuna Etkisi
}

Osman ÖZBEK*

Selçuk Üniversitesi Ziraat Fakültesi Tarım Makineleri ve Teknolojileri Mühendisliği Bölümü, Konya, Türkiye

*Sorumlu yazar: ozbek@selcuk.edu.tr

Geliş Tarihi: 16.11.2018

Düzeltme Geliş Tarihi: 08.02.2019

Kabul Tarihi: 13.02.2019

Özet

Bu çalışma tipik bir sulama amaçlı derin kuyu modeli üzerinde yürütülmüştür. Çalışmada hidrolik yük, teçhiz borusu çapı filtre tipi, filtre uzunluğu ve pompa tipi sabit tutulmuştur. Bu çalışmada sulama amaçlı derin kuyu donanımlarından çakıl zonu genişliğinin üç farklı seviyesi için, değişik pompa debisi ve dalma derinliklerinde gürültü seviyesi, çıkış basıncı, vakum basıncı, su giriş hızları, çekilen güç değerleri ölçülmüştür. Elde edilen ölçümler sonucunda farklı çakıl zonu genişliklerinin kritik dalma derinliğine, vorteks dalma derinliği ve tipine, gürültü ve güç değerlerine etkisi belirlenmiştir. Araştırma bulgularına göre her üç çakıl zonu genişliği için de debi arttıkça kritik dalma derinliği artmıştır. Kritik dalma derinliği, sabit debide ve farklı çakıl zonu kalınlıklarında belirgin bir değişim göstermemiştir. Kritik dalma derinliği tüm kombinasyonlar da 257.6 ile $617.7 \mathrm{~mm}$ arasında değişmiştir. Düşük dalma derinliklerinde oluşan vorteks dalma derinliği genel olarak debinin artması ile artmıştır. Vortekslerin tipleri debi ve dalma derinliğine bağlı olarak değişim göstermiştir. Genel olarak düşük dalma derinliklerinde sürekli hava girişli vorteks oluşurken, biraz daha yüksek dalma derinliklerinde oluşan vorteksler hava girişi olmayan tip olarak tespit edilmiştir. Pompanın sabit debi değerlerinde farklı dalma derinliklerinde oluşturduğu gürültü seviyelerinin ortalamaları en düşük ÇZK1 kombinasyonunda en yüksek ise ÇZK3 kombinasyonunda elde edildiği görülmüştür. Çakıl zonu genişliklerinin pompa kritik dalma derinliğine doğrudan etkisinin olmadığı, ancak dolaylı olarak etkilediği belirlenmiştir.

Anahtar kelimeler: Sulama derin kuyusu, çakıl zonu genişliği, pompaj, dalma derinliği, kritik dalma derinliği.

\section{Effect of Gravel Zone Widths on Critical Plunge Depth and Vortex Formation in Irrigation Wells}

\begin{abstract}
This study was carried out on a deep well model for typical irrigation purposes. In this study, hydraulic load, equipment diameter, filter type, filter length and pump type are kept constant. In this study, noise level, outlet pressure, vacuum pressure, water inflow velocity, drawn power values were measured for three different levels of gravel zone width for different irrigation flow rates and plunge depths. As a result of the measurements obtained, the effect of different pebble zone widths on critical plunge depth, depth and type of vortex plunge, noise and power values were determined. According to the research findings, the depth of the critical plunge increased as the flow rate increased for all three gravel zone widths. The critical depth of plunge did not show a significant change in constant flow and different gravel zone thicknesses. The critical depth of the plunge ranged from 257.6 to $617.7 \mathrm{~mm}$ in all combinations. The depth of the plunge plunging at the low plunge depth was increased with the increase of the flow rate. The types of vortexes vary depending on the flow and depth of plunge. Generally, vortex with continuous air inlet at low depths, while vortices formed at slightly higher plunge depths have been identified as non-air inlet type. It was observed that the average noise level of the pump at different depths of flow in the constant flow rate
\end{abstract}


values was obtained at the lowest ÇZK1 combination and the highest in the ÇZK3 combination. It was determined that the gravel zone widths had no direct effect on the critical depth of the pump but indirectly affected.

Key words: Irrigation deep well, gravel zone width, pumping depth, depth of plunge, critical depth of plunge.

\section{Giriş}

Derin kuyu pompalarının amaca uygun kullanılabilmesi için uygun tasarlanmış ve donatılmış derin kuyulara ihtiyaç bulunmaktadır. Günümüzde tarımsal amaçlı derin kuyular, sondaj yoluyla değişik çaplarda açılmaktadır. Açıklan kuyu iç kısmının akifere karşlık gelen yüzeyine filtreli borular diğer kısımlarına ise kapalı tip teçhiz boruları yerleştirilir. Filtreli teçhiz borusu dış kısmı ile kuyu iç cidarı arası çakılla doldurulup (çakıl zonu), belirli bir süre su pompajı yapılmak suretiyle yıkanarak geliştirilmektedir.

Kuyu dinamik su seviyesi, akiferin (su taşıyan jeolojik formasyon) kalınlığına, yapısına, tipine, kuyunun filtreli boru teçhiz borusunun tipine, uzunluğuna ve yerine, kuyunun çakıl zonu genişliği ve çakı özeliklerine, kuyunun yıkanması ve geliştirilmesi gibi etkenlere bağlı olarak değişim gösterebilmektedir.

Kuyu yapısı, iki ana elementten oluşmaktadır. Bu elementlerden birisi, kuyu pompa ekipmanının yer alacağı iç bölümdür. Bu bölüm kuyunun borulanmış bölümüdür. Bir diğer bölüm ise, kuyuya, su girişinin olduğu, bölümdür (çakıl zonu). Akiferden gelen su, bu bölümden geçerek kuyuya, gireceğinden, bu bölüm kuyu performansını direkt olarak etkilemektedir. Bu nedenle, bu bölümün oluşturulmasına ilişkin parametrelerin çok dikkatli seçilmesi ve akifer parametrelerini olumsuz etkilememesine özen gösterilmesi gerekmektedir (Çebi, 1994).

Çakıl, akiferi desteklemek, delik geçirgenliğini artırmak veya akiferdeki materyalin filtrelenmesine yardımcı olmak için filtrenin dışına yerleştirilir. Yapay filtre olarak hizmet ettiğinden çakıl materyalinin boyut dağılım seçiminin önemi büyüktür (Rafferty, 2001). Çakıl malzemesinin tane büyüklüğü ve akifer kum tanelerinin büyüklüğü arasındaki ilişki uygunsuzluğu kuyu çakıl paketinden kumun pompalanmasına neden olur.

Çakıl zonunda kullanılacak malzemenin niteliği ve boyutu yanında, çakıl zonunın kalınlığı da dikkate alınmalıdır (Çebi, 1994). Kuyu veriminin düşmesinin en önemli etkilerden biride çakıl zonunun daha az geçirgenliğidir (Polak ve ark., 2016). Çakıl zonunun kalın olması (geçirgenliğinin az olması) kuyunun verimini artırmamasının yanında formasyon kumunun kuyuya girmesini önleme gibi bir etkisi de olmamaktadır. Formasyon kumunun kuyuya girmemesi için, formasyon materyali ile çakıl zonunda kullanılan malzemenin tane boyutlarının uygunluğudur (Boman ve ark., 2003; Çebi, 1994).

Çakıl zonu kuyu cidarında oluşan enerji miktarını düşürmektedir. Çakıl zonunun ince tutulması kuyu geliştirme esnasında istenmeyen ince kum, silt ve kilin temizlenmesini kolaylaştırmaktadır (Akpınar, 1999). Literatürde çakıl zonunın kalınlığının genellikle $7.5 \mathrm{~cm}$ ile $20 \mathrm{~cm}$ arasında olması gerektiği bildirmişlerdir (Akpınar, 1999; Çebi, 1994; Rafferty, 2001; Boman ve ark., 2003).

Pompaya su girişi ile su yüzeyi arasındaki kalan dikey mesafeye dalma derinliği (S) denilmektedir (Şekil 1). Dalma derinliği, kritik dalma derinliği $\left(S_{k}\right)$ değerden küçük olması durumunda vorteks oluşmaktadır. Bunun sonucunda pompa emişi kaybetmekte ve verimde azalmalar görülmektedir (Yildirim ve ark., 2011; Sarkardeh, 2017).

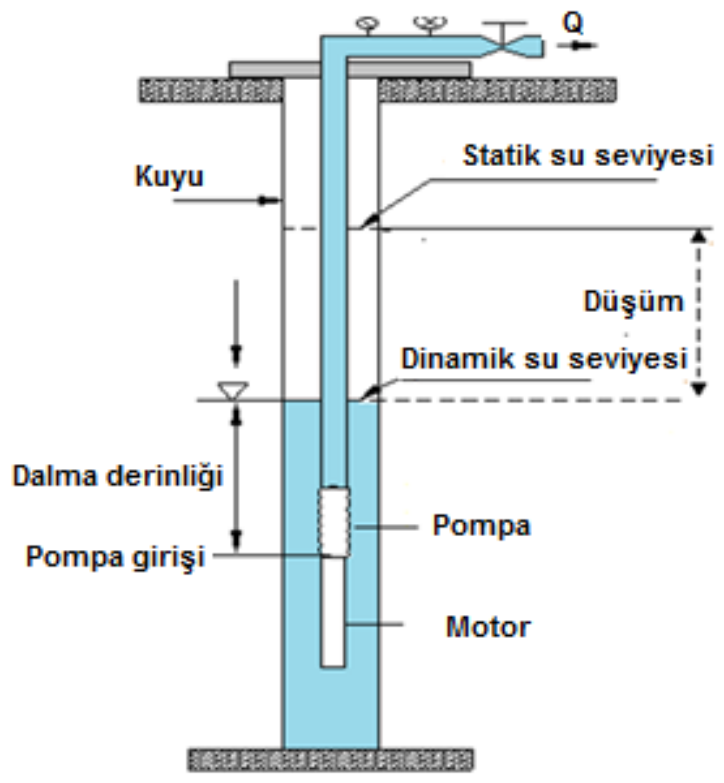

Şekil 1. Derin kuyu temel yükseklik terimleri (Çalışır, 2009).

Bu çalışmada sulama amaçlı derin kuyu donanımlarından çakıl zonu genişliğinin pompada kritik dalma derinliğine ve vorteks oluşumuna etkisi incelenmiştir. Bu bağlamda farklı çakıl zonu genişliklerinin, değişik debi $(Q)$ ve dalma derinlikleri (S) seviyelerinde pompa gürültü değerleri (G), şebekeden çekilen güç $(\mathrm{N})$, pompa çıkış $(\mathrm{Pb})$ ve vakum 
basıncı (Pe), sıcaklıklar ( $T$ ), bağıl nem (BN) gibi değerler ölçülmüştür. Ayrıca ölçülen bazı değerlerin faklı dalma derinlikleri seviyelerinde ve vorteks oluşum durumlarında değişimleri de incelenmiştir. Düşük dalma derinliklerinde ortaya çıkan vorteksler görüntülenmiş ve sınıflandırılmıştır.

\section{Materyal ve Yöntem \\ Materyal}

Denemeler Selçuk Üniversitesi Ziraat Fakültesi

Tarım Makineleri ve Teknolojileri Mühendisliği
Bölümü Prof. Dr. Şinasi YETKIN Tarım Makineleri ve Teknolojileri Mühendisliği Uygulama Atölyesinde Derin Kuyu Test Ünitesinde yapılmıştır (Şekil 2).

Pompa test kulesinde bulunan ve pompa denemelerinde kullanılan ölçme enstrümanlarının bazı teknik özellikleri Çizelge 1'de verilmiştir.
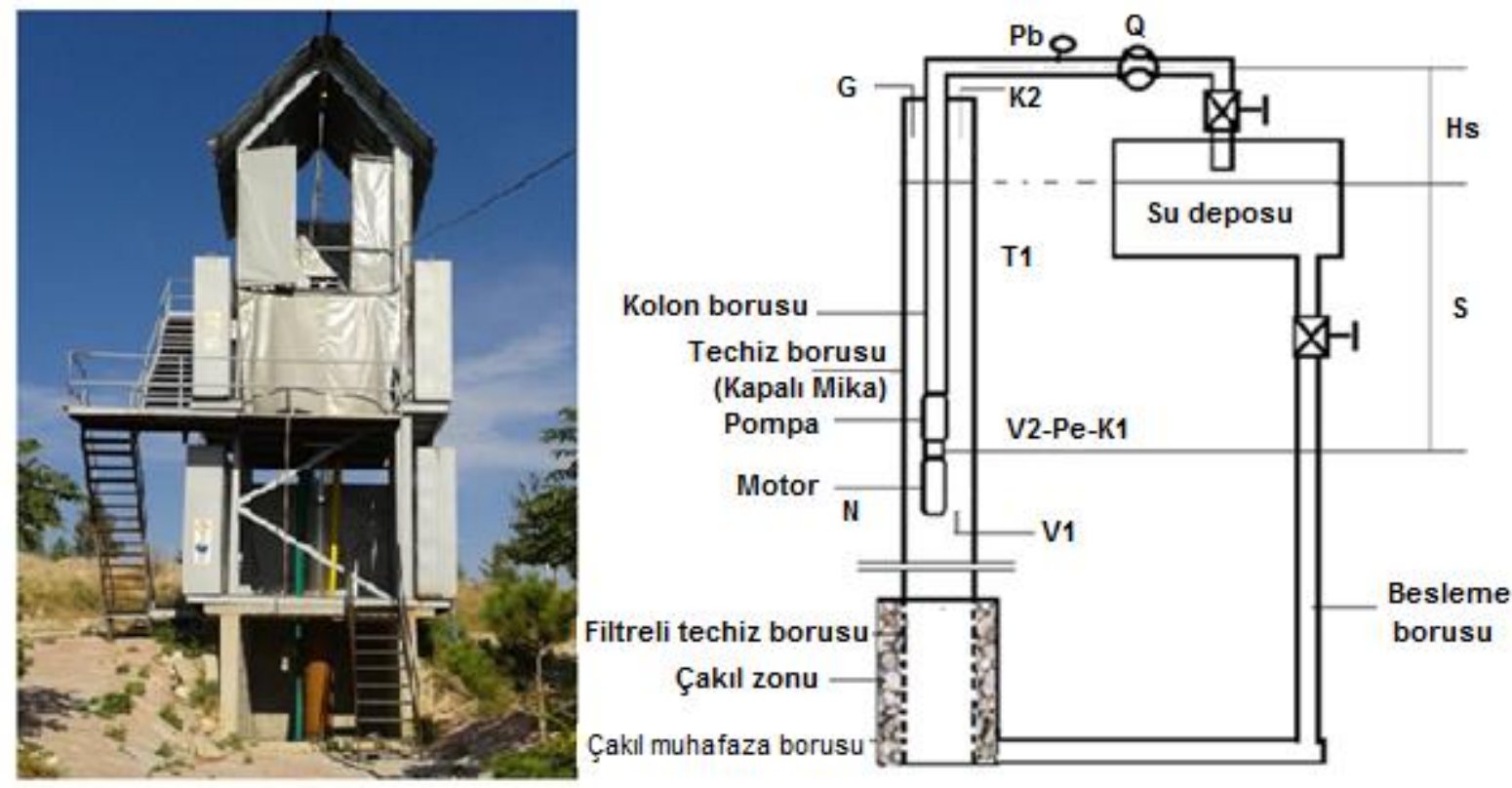

Şekil 2. Derin kuyu test ünitesi ve cihazların bağlantı yerleri.

Çizelge 1. Kullanılan ölçme aletlerinin bazı teknik özellikleri

\begin{tabular}{|c|c|}
\hline Ekipman cinsi & Bazı teknik özellikleri \\
\hline Pompa & Anma çapı 6",2 kademeli, mil çapı 25 mm, Radyal çark, 4.5 mm klerens açıklığı \\
\hline Elektrik motoru & $\begin{array}{l}\text { Suver, } 380 \text { V, } 8.2 \text { A, } 50 \text { Hz, } 2869 \text { 1/min, } 4.5 \text { kW, mil çapı: } 25 \text { mm, Su soğutmalı, 3x2.5mm² } \\
\text { kablo kesiti }\end{array}$ \\
\hline Debimetre & $\begin{array}{l}\text { S MAG } 100 \text { TiP, DN 80/HR/316 flanş bağlantılı elektromanyetik debimetre, } 220 \text { V beslemeli } \\
\text { dijital göstergeli, anlık debi, yüzde akış ve toplam gösterimli. Ayarlanabilir 4-20 m/A plus ve } \\
\text { frekans çıkışlı. Çalışma debisi } 1-280 \mathrm{~m}^{3} / \mathrm{h} \text {, çalışma basıncı } 16 \text { bar. }\end{array}$ \\
\hline Manometre & WikA, 0-10 bar, Alltan Bağlantılı, 4-20 mA çıkışlı. \\
\hline Seviye ölçer & Hydrotechnik marka, 010 tip/1.5 V, 150 m’lik ölçeklendirilmiş kablolu, ses ve ışık ikazlı tip. \\
\hline Sıcaklık sensörleri & Turck marka, $10-24$ VDC, $-50 \ldots 100^{\circ} \mathrm{C}, 4-20 \mathrm{~mA}$ output. \\
\hline Noise Sensor & $\begin{array}{l}\text { CT-2012 model, input } 4 \mathrm{~mA}, \mathrm{DC} 24 \mathrm{~V} \text { power supply output indicator. Sound Level } \\
\text { Transmitter Model : TR-SLT1A4, Measurement range:30-80 dB, 50-100 dB, } 80-130 \mathrm{~dB} \text {, } \\
\text { output 4-20 mA, } 90-260 \mathrm{ACV} 50 \mathrm{~Hz} / 60 \mathrm{~Hz} \text {, Operation temperature } 0-50{ }^{\circ} \mathrm{C} \text {. }\end{array}$ \\
\hline Camera & $\begin{array}{l}\text { Radial axis camera; } 1080 \text { p HD Sensor, } 720 p \text { HD video } \\
\text { Axial axis camera; } 15.0 \text { megapixels, Full HD video recording }\end{array}$ \\
\hline Bilgisayar & Asus intel core i7. \\
\hline
\end{tabular}


Denemede farklı çakıl muhafaza boruları için yaklaşık $2 \mathrm{~m}^{3}$ civarında temin edilen temiz ve yıkanmış çakıl kullanılmıştır. Kullanılan çakılın yığın haldeki görünümü Şekil 3'te verilmiştir.

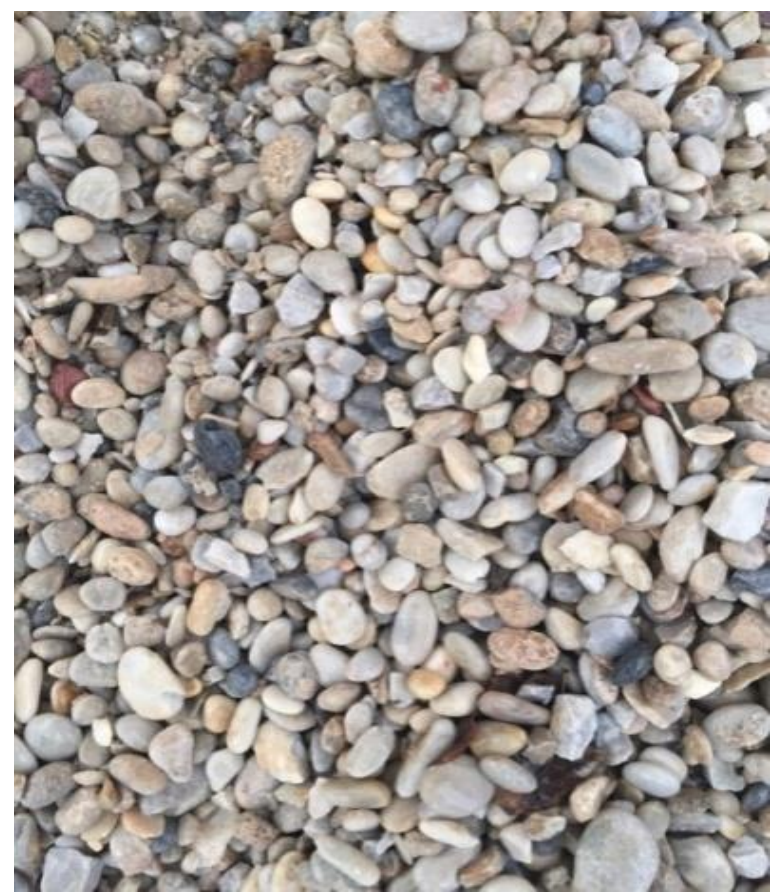

Şekil 3. Deneylerde kullanılan çakılın yığın haldeki görünümü.

Çakıl yığını içinden gelişi güzel alınan 100 adet çakıl örneği üzerinde yapılan ölçümlerin sonucunda belirlenen çakıla ait bazı fiziksel özelliklerinin ortalamaları Çizelge 2'de verilmiştir.

Çizelge 2. incelendiğinde çalışmada kullanılan çakılın \%76'sının 7-15 $\mathrm{mm}$ aralığında olduğu belirlenmiştir.

Negatif basıncı (vakum) emiş ağzı alt adaptörüne yerleştirilen cıvalı bir $U$ diferansiyel basınçölçer ile ölçülmüştür. $U$ diferansiyel basınçölçer için $6 \mathrm{~mm}$ çapında, 2 m uzunluğunda şeffaf hortum ve $200 \mathrm{~g}$ cıva kullanılmıştır. Negatif basınçölçerin bağlantısı Şekil 4 'te verilmiştir.

\section{Yöntem}

Test düzeneği tabandan itibaren 12"çapa sahip $2 \mathrm{~m}$ uzunluğunda dikey oblong delikli filtreli teçhiz borusu, $4 \mathrm{~m}$ kapalı teçhiz borusu ve $4 \mathrm{~m}$ şeffaf teçhiz borusu denemeler sırasında sabit tutulmuştur Çakıl zonu filtrenin etrafına özel hazırlanmış çakıl muhafaza borularının yardımı ile doldurulmuştur (Şekil 5). Pompa işletme karakteristiklerinin ölçülmesinde ve yapılan hesaplamalarda TS EN ISO 9906 standardı, gürültü ölçümlerinde ise EN ISO 3740 standardı esas alınmıştır (Anonim, 2002; 2014).

Çakıl zonu kalınlığı ÇZK1, ÇZK2 ve ÇZK3 olmak üzere üç seviyede oluşturulmuştur. ÇZK1, ÇZK2 ve ÇZK3 çakıl zonu kalınlıkları sırasıyla $5 \mathrm{~cm}, 10 \mathrm{~cm}$ ve $15 \mathrm{~cm}$ değerindedir.

Çizelge 2. Deneylerde kullanılan çakılın bazı fiziksel özellikleri ortalama değerleri

\begin{tabular}{lc}
\hline Fiziksel özellik & $\begin{array}{c}\text { Ortalama } \\
\text { değeri }\end{array}$ \\
\hline Hacım ağırı̆̆ı $\left(\mathrm{kg} \mathrm{dm}^{-3}\right)$ & 1.54 \\
Parçacık yoğunluğu $\left(\mathrm{kg} \mathrm{dm}^{-3}\right)$ & 2.75 \\
Porozite (\%) & 44 \\
Parçacık genişliği (mm) & 14.3 \\
Parçacık uzunluğu (mm) & 19.6 \\
Parçacık kalınlığı (mm) & 9.1 \\
Parçacık eşdeğer geometrik çapı & 13.5 \\
(mm) & 70 \\
Parçacık küreselliği (\%) & 22.76 \\
Doğal yığılma açısı ( ${ }^{\circ}$ & 41.9 \\
Metal-Çakıl statik sürtünme & \\
katsayısı (-) & \\
Parçacıkların geometrik çap bakımından \% \\
frekans dağılımı & 8 \\
\hline 7.68 mm (min.) -10.00 mm (\%) & 46 \\
10.01 mm -13.50 mm (\%) & 22 \\
13.51 mm -15.00 mm (\%) & 12 \\
15.01 mm -18.00 mm (\%) & 12 \\
18.01 mm-21.94 mm (max.) (\%) & \\
\hline
\end{tabular}

Çakıl zonu genişliklerinin her birinde dalgıç pompa (D) optimum çalışma devrinde, 5 değişik debi aralığının (40-45-50-55-60 $\left.\mathrm{m}^{3} / \mathrm{h}\right)$ her biri için 4-7 değişik dalma derinliklerinde ölçümler alınmıştır (Şekil 6.). Pompa belirlenen herhangi bir debi değerinde çalıştırılarak ilk değerler kayıt altına alındıktan sonra dalma derinliği düşürülmüştür. Dalma derinliğinin düşmesi ile değişen debi değeri ölçme borusunda bulunan vana ile tekrar eski haline getirilmiştir. Bu şekilde bir debi değerinde 4-7 farklı dalma derinliklerinde ölçümler kayıt edilmiştir.

Çalışmada ölçülen büyüklüklerin kaydedilmesi için yazılım ve otomasyon sistemi gerçekleştirilmiştir. Bu sistemin blok diyagramı Şekil 7'de verilmiştir. Blok diyagramından da görüldüğü gibi sistemde bulunan sensörlerden alınan bilgiler merkezi bir veri toplama kartı üzerinden kablosuz (Bluetooth) olarak Bilgisayar'a aktarılmaktadır. Merkezi işlemcide depolanan bilgiler bilgisayarda hazırlanan yazılım ara yüzü aracılığı ile operatör tarafından istenilen aralıklarda uygun isimlerle kayıt edilmektedir. Kayıt etme işlemi, saniyede bir adet verileri alabilecek 
tarzda hazırlanmıştır. Pompa rejime girdikten sonra kayıt işlemine başlanılmış ve bir sensör den 50 adet veri alınmıştır. Alınan bu verilerin ortalamaları ve standart hataları çizelge olarak verilmiştir. Grafiklerin çiziminde, deneyler sırasında elde edilen ham rakamların hesaplanması ve standart hatalarının belirlenmesinde Excel paket programları kullanılmıştır.

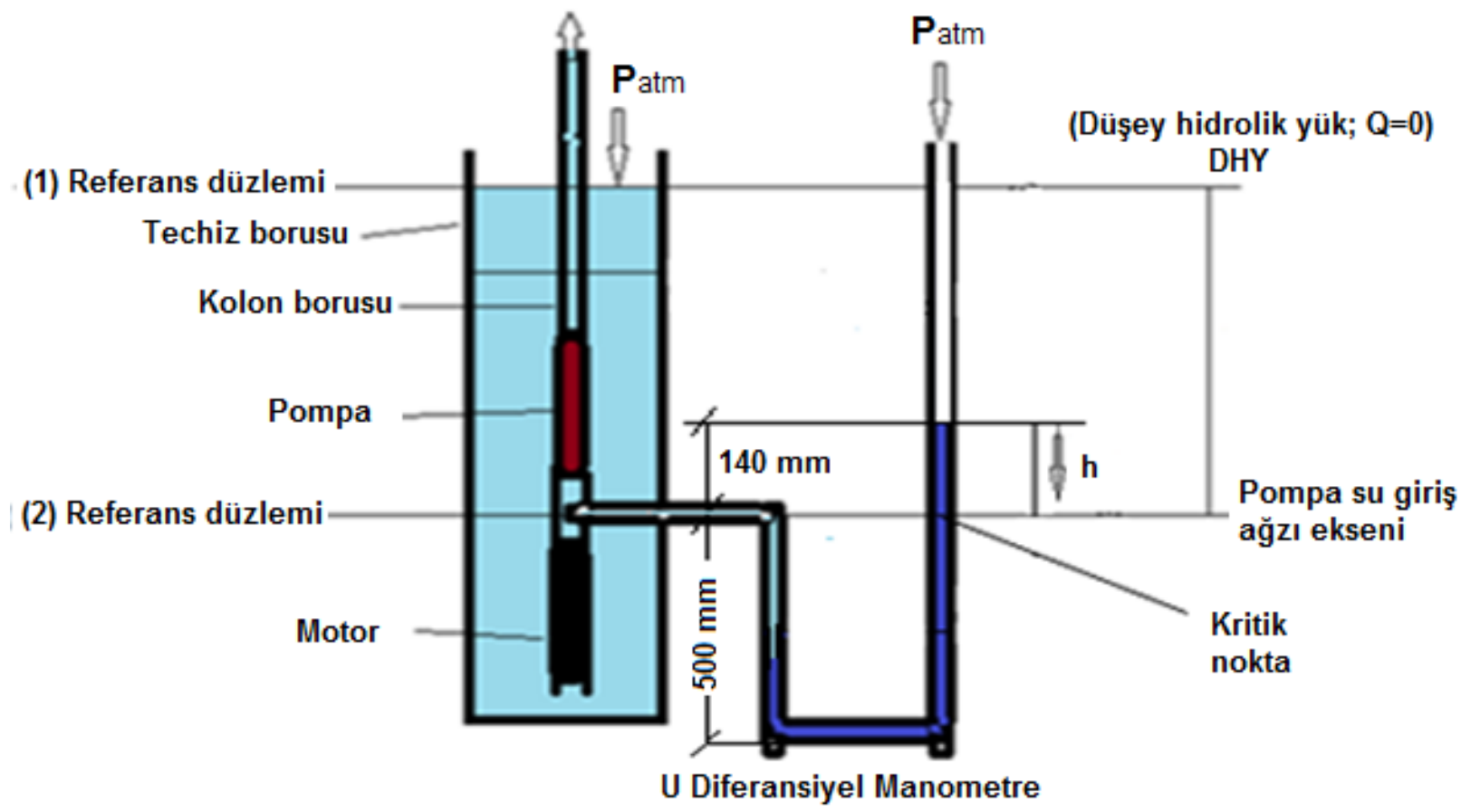

Şekil 4. U Diferansiyel tipi negatif basınçölçer.

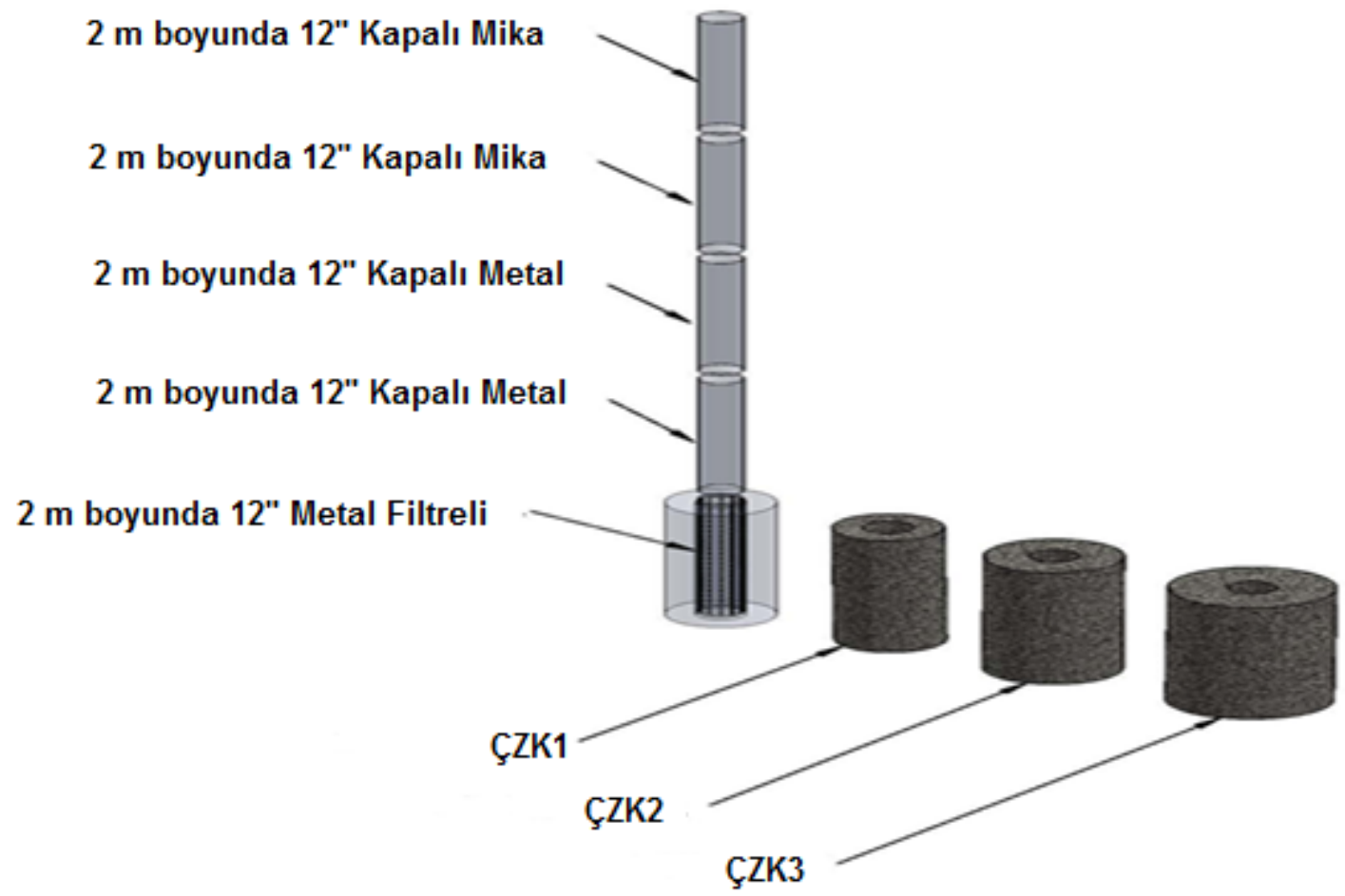

Şekil 5. Çakıl zonu kalınlığı için kuyu donanımı. 


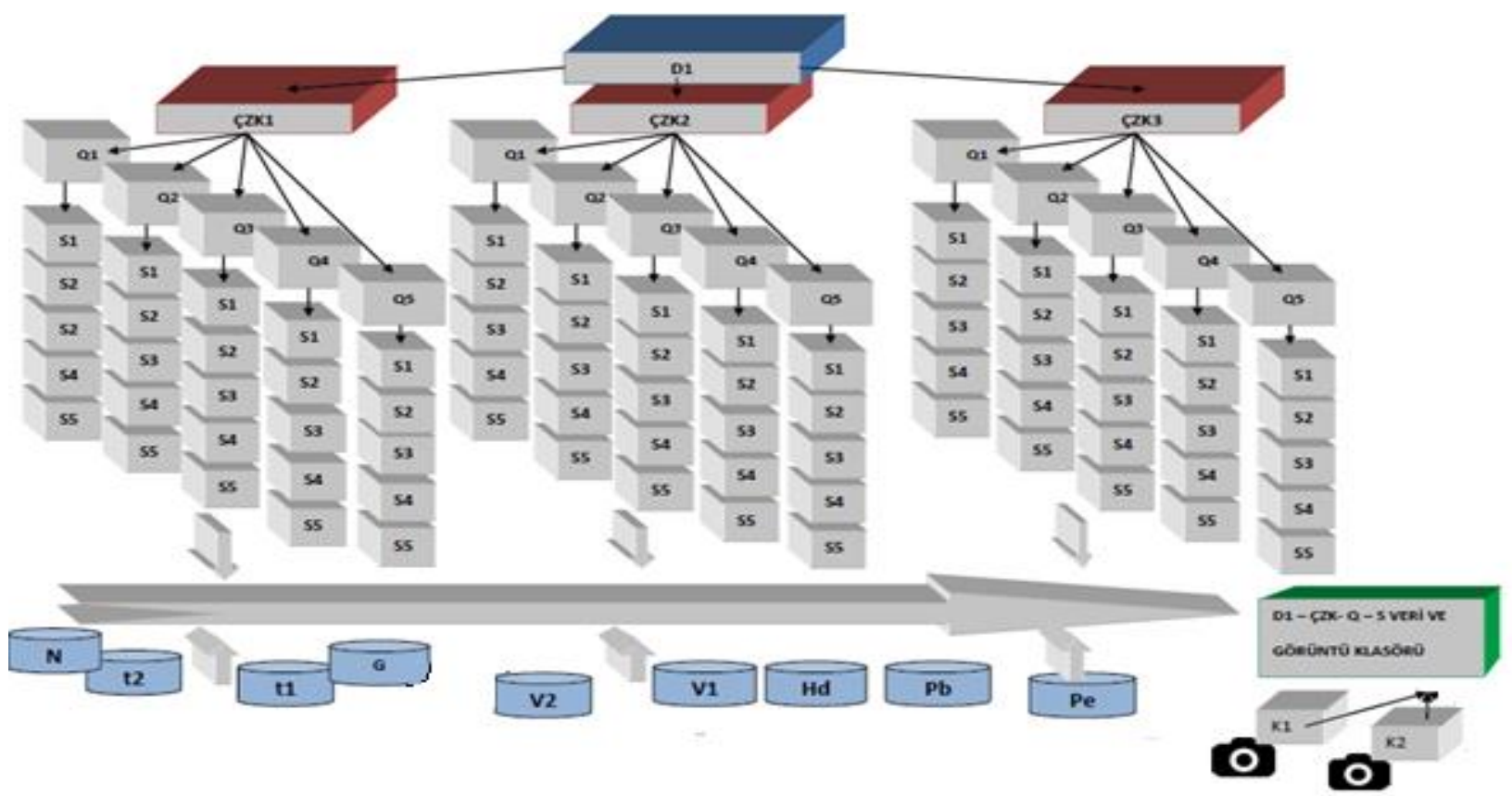

Şekil 6. Deneme planı.

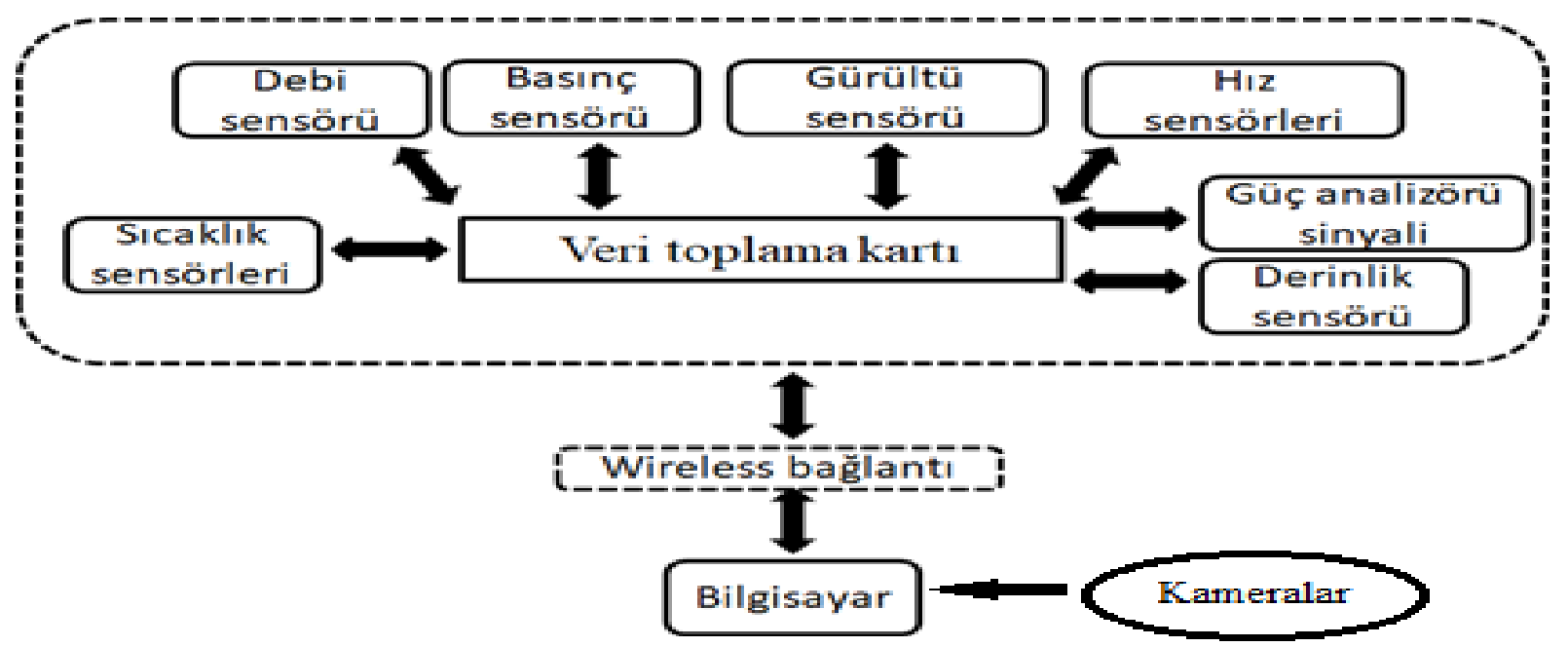

Şekil 7. Otomasyon sisteminin blok diyagramı.

Pompa $1880 \mathrm{~mm}$ dalma derinliğinde (sabit hidrolik yük) denemeler yürütülmüştür. Seviye ölçer ile düşüm ölçülerek aşağıdaki eşitlikle dalma derinliği hesabı yapılmıştır.

$\mathrm{S}=1880-\Delta$ $(\mathrm{mm})^{\prime}$ dür.

Burada $\mathrm{S}=$ Dalma derinliği $(\mathrm{mm})$ ve $\Delta=$ Düşüm

Pompa emiş basıncı, pompa giriş ağzına monte edilen cıvalı $U$ diferansiyel monometre ile belirlenmiş ve kayıtlanmıştır. Şekil 3'de gösterildiği üzere cıvalı U diferansiyel manometresinin sağ kolundaki cıva seviyesi ile 2 no'lu referans düzlemi arasındaki yükseklik $h$ ölçülmüş ve kaydedilmiştir. Pompa çalıştırılmadan önce $h$ yüksekliği düşey hidrolik yüke eşittir ve dalma derinliği maksimum değerdedir. Pompa çalıştırıldığında çekilen debiye bağlı olarak emiş arttıkça $h$ yüksekliği azalmaktadır. $h$ değerinin sıfırdan sonrası pompa emişinde negatif basınç oluşmaktadır. Teçhiz borusundaki pozitif su yükü pompa emiş ağzında geliştirilen emiş yükünü karşılayamamaktadır. Devam edecek vakum artışı ile emiş ağzından hava girmesine neden olacaktır. $h$ değerleri mm olarak en az üç tekerrürlü ölçülmüş ve kaydedilmiştir. Ölçülen $h$ değeri dikkate alınarak pompanın emiş ağzı basıncına aşağıdaki eşitlik yardımıyla dönüştürülmüştür.

$$
\mathrm{Pe}=\left[\rho^{*} \mathrm{~g}^{*}(\mathrm{~h} / 100)\right] / 1000 \quad(\mathrm{kPa})
$$


Burada cıvanın yoğunluğu $\rho c=13600 \mathrm{~kg} \mathrm{~m}^{-3}$ ve yer çekimi ivmesi $\mathrm{g}=9.81 \mathrm{~m} \mathrm{~s}^{-2}$ olarak kabul edilmiştir.

Sabit debilerde, dalma derinliğinin fonksiyonu olarak Pe ilişkisinden elde edilen regresyon denklemlerinden Pe değerini sıfır kılan dalma derinliği (S) değeri hassas olarak belirlenmiş ve kritik dalma derinliği (Sk) olarak kabul edilmiştir.

Denemelerde, deney sisteminin iki ayrı noktasından akış hızı ölçülmüştür (Şekil 2). Pompa kolon borusu içinde ortalama su akış hızı $\left(v_{1}\right)$, kuyu teçhiz borusu ile pompa anma çapı arasında kalan

Vorteks tipleri
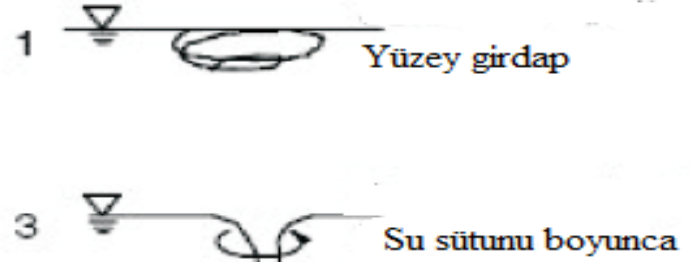
tutarl girdap

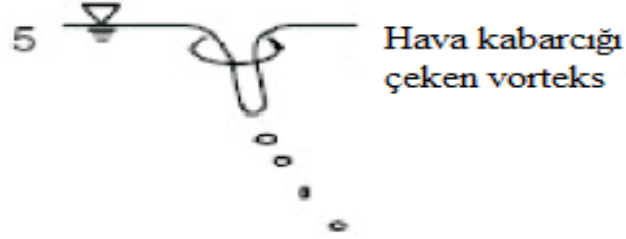

Şekil 8. Vorteks tipi sınıflandırılması (Anonim, 1998).

\section{Bulgular ve Tartışma Çakıl zonu kalınlığının kritik dalma derinliğine etkisi}

Denemelere, $188 \mathrm{~cm}$ sabit hidrolik yük altında $89 \mathrm{~cm}$ statik su seviyesinde başlanmıştır. 40, 45, 50, 55 ve $60 \mathrm{~m}^{3} / \mathrm{h}$ debi değerlerinde yapılan ölçümler sonucunda üç ayrı çakıl zonu genişliği için elde edilen veriler Ek Çizelge 1, 2 ve 3 'de verilmiştir.

Çakıl zonu kalınlıklarının sabit debi değerlerinde dalma derinliğinin fonksiyonu olarak Pe değerleri Şekil 9,10 ve $11^{\prime}$ de verilmiştir. Bu fonksiyonlardan regresyon denklemleri çıkartılarak Pe basınç değerinin sıfır olduğu kritik dalma derinliği seviyeleri hesaplanmış ve Çizelge 3'de verilmiştir.

Şekil 11'de görülen $55 \mathrm{~m}^{3} \mathrm{~h}^{-1}$ debideki S-Pe ikinci bölgede oluşması pompanın bu kuyu donanımı ve debide kritik dalma derinliğinin aşıldığı, vorteksli çalışma durumunda olduğunu göstermektedir. Bu halka boşluk mesafesinin 2/3'lük kısmı kadar içeriye uzatılarak, pompa giriş ağzındaki akış hızı ( $\left.v_{2}\right)$ için sensör probu pompa emiş ağzı içine monte edilmiştir. Vorteks tiplerinin belirlenmesinde radyal (K1) ve eksenel (K2) yönlerden video görüntüleri alınarak incelenmiştir. Incelemeler sonucunda ortaya çıkan vorteks görüntülerinin tipleri Şekil 8'e göre sınıflandırılmış. Vorteks tiplerinin oluştuğu andaki dalma derinliği vorteks dalma derinliği (Sv) olarak belirlenmiştir.
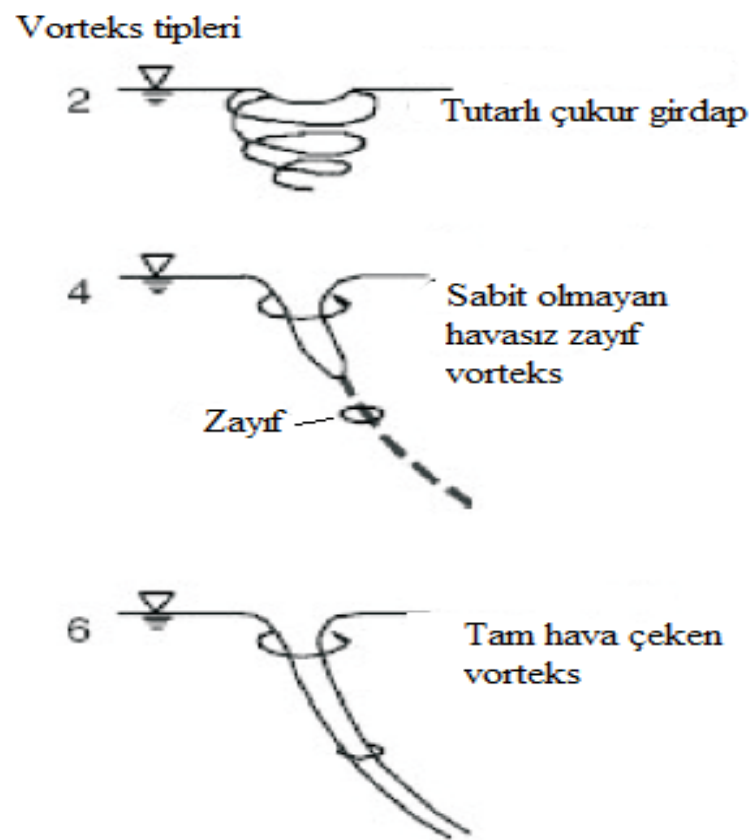

kombinasyondaki $60 \mathrm{~m}^{3} \mathrm{~h}^{-1}$ debi değeri de elde edilememiştir.

Çizelge 3 incelendiğinde sabit çakıl zonu kalınlıklarında debi arttıkça kritik dalma derinlikleri yükselmiştir. Yapılan çalışmalarda da kritik dalma derinliğinin debi ile orantılı olduğu görülmüştür ( Hanson, 2000; Christiansen, 2005; Möller ve ark., 2015; Sarkardeh, 2017).

Ancak sabit debilerde çakıl zonu kalınlığının kritik dalma derinliği üzerine etkisi görülmemiştir.

\section{Çakıl zonu kalınlığının vorteks derinliği (sv) ve vorteks tipi üzerine etkisi}

Sabit düşey hidrolik yük, farklı debi ve dalma derinlikleri ve çakıl zonu kalınlıklarında K1 ve K2 kameraları ile radyal ve eksenel yönde kaydedilen video kamera görüntüleri fotoğraflanmıştır. Fotoğraflanan görüntülerden bazıları Ek Şekil 1,2 ve 
3'de verilmiştir. Buna göre vorteks oluştuğu anda fotoğraflanan görüntülerin dalma derinlikleri vorteks dalma derinliği (Sv) olarak belirlenmiş ve vorteks tipleri tespit edilmiştir (Çizelge 4).

Çizelge 3. Çakıl zonu kalınlığı ve debi ile kritik dalma derinliği $\left(S_{k}\right)$ değişimi

\begin{tabular}{cccc}
\hline $\mathbf{Q}$ & \multicolumn{3}{c}{$\boldsymbol{S}_{\mathbf{k}}(\mathbf{m m})$} \\
\hline$\left(\mathbf{m}^{\mathbf{3}} \mathbf{h}^{\mathbf{- 1}}\right)$ & ÇZK1 & ÇZK2 & ÇZK3 \\
\hline 40 & 257.6 & 260.8 & 260.5 \\
45 & 337.2 & 324.1 & 332.2 \\
50 & 419.3 & 411.5 & 430.1 \\
55 & 503.0 & 511.8 & 503.9 \\
60 & 607.7 & 617.7 & - \\
\hline
\end{tabular}

Çizelge 4. Farklı çakıl zonu kalınlıkları ve debilerde vorteks dalma derinliği (Sv) ve vorteks tipi

\begin{tabular}{ccccc}
\hline & $\mathbf{Q}\left(\mathbf{m}^{\mathbf{3}} \mathbf{h}^{-\mathbf{1}}\right)$ & ÇZK1 & ÇZK2 & ÇZK3 \\
\hline \multirow{2}{*}{ Vorteks } & 40 & 20 & 20 & 25 \\
dalma & 45 & 55 & 25 & 20 \\
derinliği & 50 & 80 & 60 & 50 \\
$\left(\mathbf{S}_{\mathbf{v}}-\mathbf{m m}\right)$ & 55 & 30 & 50 & 80 \\
& 60 & 80 & 75 & - \\
\hline \multirow{4}{*}{ Vorteks } & 40 & 6 & 6 & 6 \\
tipi & 45 & 1 & 6 & 6 \\
& 50 & 1 & 2 & 4 \\
& 55 & 6 & 5 & 1 \\
& 60 & 4 & 4 & - \\
\hline
\end{tabular}

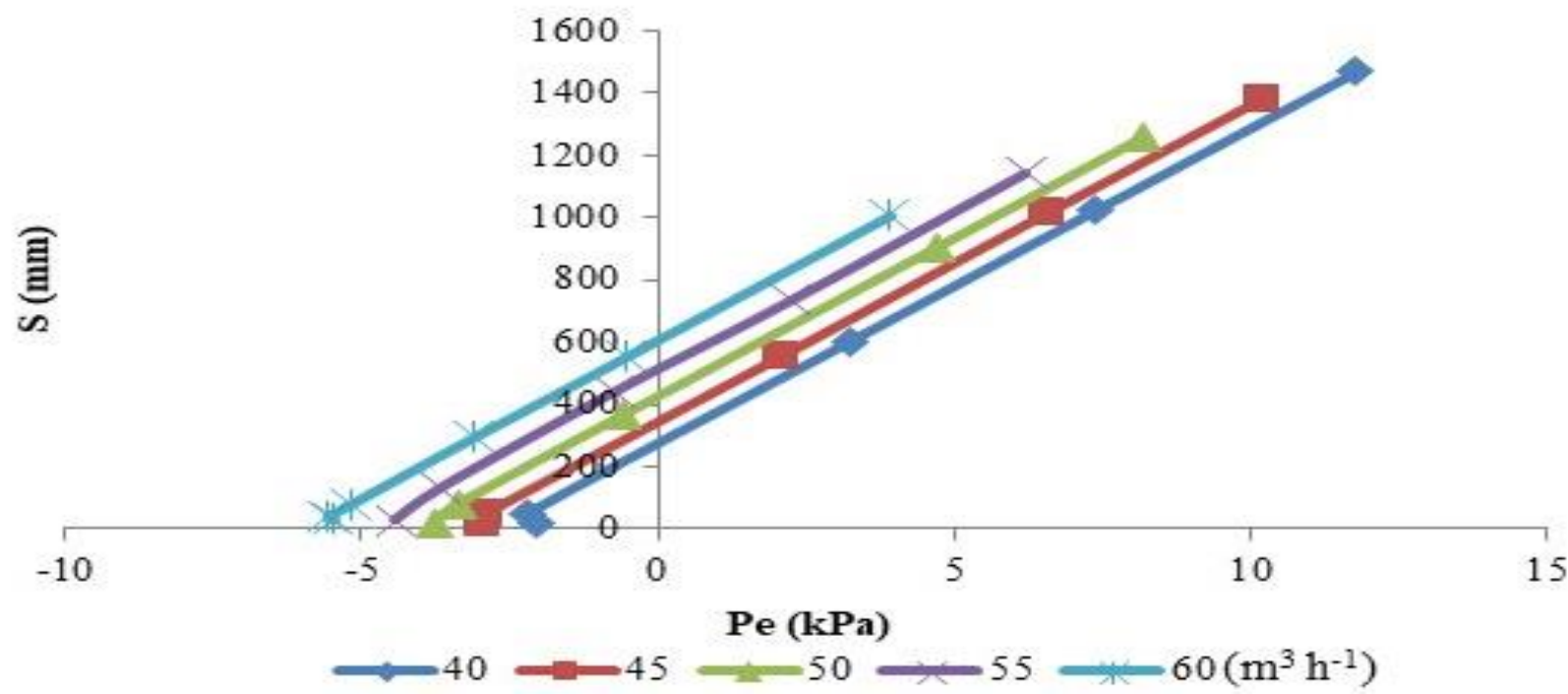

Şekil 9. ÇZK1 çakıl zonu kalınlığı ve farklı sabit debilerde S ve Pe ilişkisi.

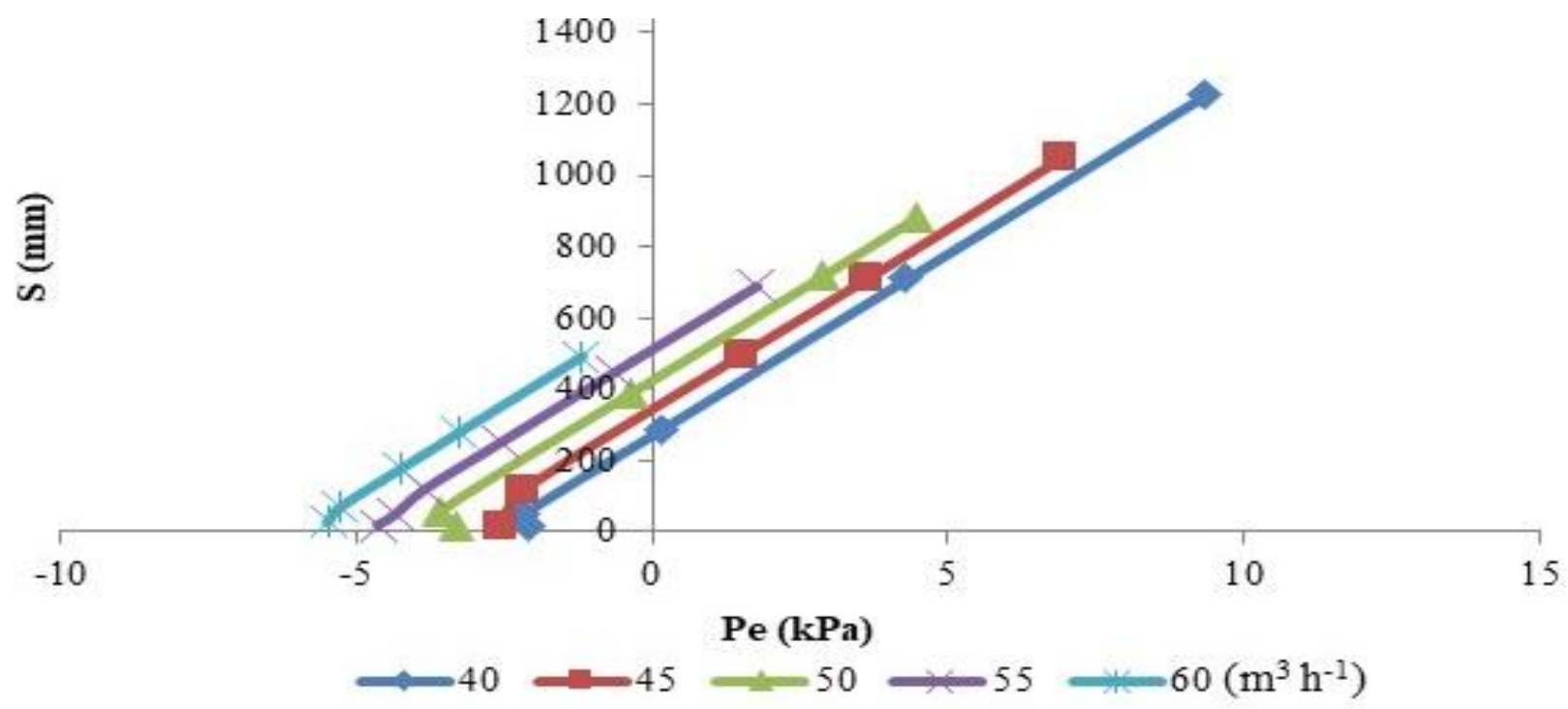

Şekil 10. ÇZK2 çakıl zonu kalınlığı ve farklı sabit debilerde S ve Pe ilişkisi. 


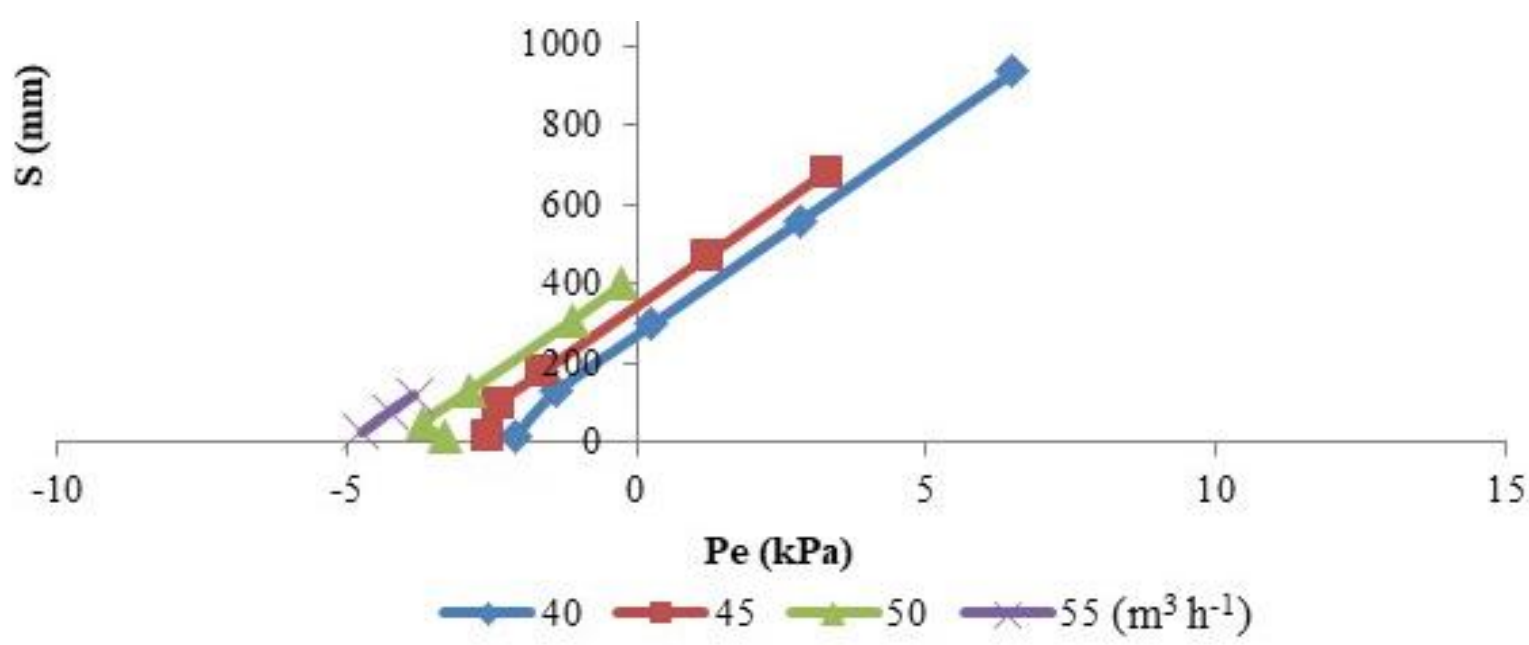

Şekil 11. ÇZK3 çakıl zonu kalınlığı ve farklı sabit debilerde S ve Pe ilişkisi.

Farklı çakıl zonu kalınlıklarının vorteks dalma derinliğine ve vorteks tipine belirgin bir etkisinin olduğu görülmemiştir. Tüm çakıl zonu kalınlıklarında debi artması ile genellikle vorteks dalma derinliği seviyesi yükselmiştir. Ancak sabit debide çakıl zonu değişimlerinin vorteks dalma derinliğine ve tipine belirgin bir etkisi görülmemiştir. Vortekslerin tamamen düşük dalma derinliklerinde oluştuğunu ve çakıl zonu kalınlıkları ile bir ilişkisinin olmadığı söylenebilir.

Çakıl zonu kalınlığının tüm seviyelerinin düşük dalma derinliklerinde $(20-40 \mathrm{~mm})$ sürekli hava girişli vorteks olduğu belirlenmiştir. Bu oluşan vorteksin ise tip 6'ya girdiği saptanmıştır. Okamura ve ark. (2007) yaptıkları çalışmalarında düşük dalma derinliğinde oluşan vorteksi sürekli hava çeken tip olarak belirtmişlerdir. Yüksek dalma derinliklerinde (30-100 $\mathrm{mm}$ ) oluşan vorteks tipini ise hava girişi olmayan tip 34-5 olarak sınıflandırabiliriz.

Çizelge 3 ve 4'ü incelediğimizde kritik dalma derinliği ile vorteks dalma derinliği seviyeleri arasında fark olduğu görülmektedir. Bunun sebebini, pompa giriş ekseninde oluşan hidrolik yükün azalması ve pompa vakum basıncının artması ile aradaki basınç farkının yükselmesine bağlayabiliriz. Basınç farkının yükselmesi düşük dalma derinliklerinde suya bir hareket kazandırmıştır. Bu hareket sonucunda da vorteksler oluşmuştur.

\section{Farklı çakıl zonu kalınlıklarında dalma derinliği değişiminin gürültü ve çekilen güç üzerine etkisi}

Ek Şekiller 1,2 ve 3 incelendiğinde pompanın sabit debi değerlerinde farklı dalma derinliklerinde oluşturduğu gürültü seviyelerinin ortalamaları en düşük 74.01 dBA ile ÇZK1 kombinasyonunda $50 \mathrm{~m}^{3} \mathrm{~h}^{-1}$ debi değerinde elde edilirken, en yüksek ise $78.83 \mathrm{dBA}$ ile ÇZK3 kombinasyonunda $55 \mathrm{~m}^{3} \mathrm{~h}^{-1}$ debi değerinde elde edildiği görülmüştür. ÇZK1 kombinasyonda $50 \mathrm{~m}^{3}$ $\mathrm{h}^{-1}$ debi değerinde gürültü seviyesi ortalamasının en düşük çıkmasının sebebi, çakıl zonu kalınlığının az olması ve pompanın optimum debi aralığında (50-55 $\mathrm{m}^{3} \mathrm{~h}^{-1}$ ) çalışmasına bağlayabiliriz. Çalışır ve ark. (2007), yaptıkları araştırmasında farklı yatay milli santrfüj pompaların optimum debi değerlerinde en düşük gürültü değerlerinin elde edildiğini bildirmişlerdir. ÇKZ3 kombinasyonun da gürültü seviyesi ortalamasının yüksek çıkmasının sebebini ise çakıl zonu kalınlığının fazla olması doğrudan düşümü arttırdığı için pompa tamamen kritik dalma derinliğinin altında çalışmasına bağlayabiliriz.

Çakıl zonu kalınlıklarında pompanın farklı debi değerlerinde oluşan gürültü seviyelerinin dalma derinliğine bağlı değişimi Şekil 10'da verilmiştir.

Dalma derinliği değişimlerinde gürültü seviyelerinin genellikle sabit bir seyir izlediği Şekil 10’da görülmektedir. Çakıl zonu kalınlıklarının tüm kombinasyonların da düşük dalma derinliklerinde oluşan vorteksin etkisi ile pompaya giren hava gürültü seviyesi değerlerini ani olarak yükseltmiştir. Pompaya hava girmesiyle kavitasyon oluşmuştur. Čdina (2003), yaptığı çalışmasında pompanın kavitasyonlu durumda oluşan gürültü seviyelerinin $147 \mathrm{~Hz}$ frekans 70-80 dBA olarak belirtmiştir. $\mathrm{Bu}$ gürültü seviyesinin kavitasyonsuz çalışma durumuna göre belirgin şekilde ayrıştığını ve gürültü seviyesi ölçümlerinden kavitasyon tahmini yapılabileceğini açıklamıştır.

Çakıl zonu kalınlıklarında pompanın farklı debi değerlerinde çektiği gücün dalma derinliğine bağlı değişimi Şekil 11'de verilmiştir. 
Türk Tarım ve Doğa Bilimleri Dergisi 6(2): 153-167, 2019

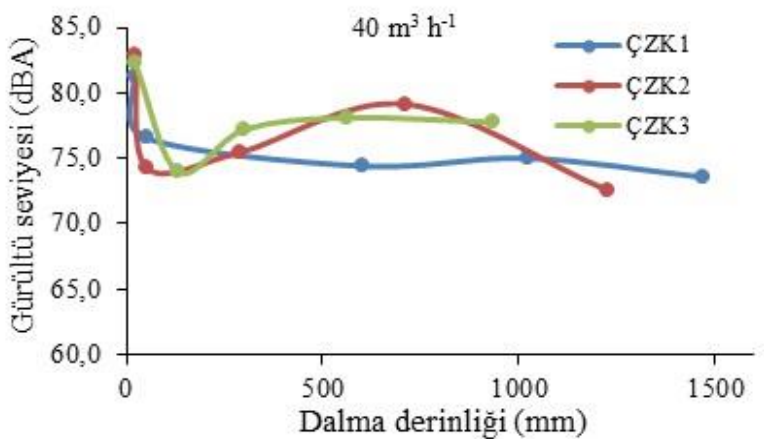

(a)

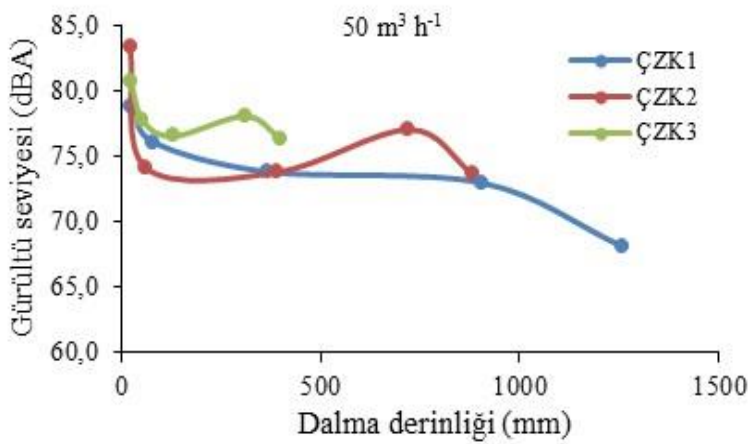

(c)

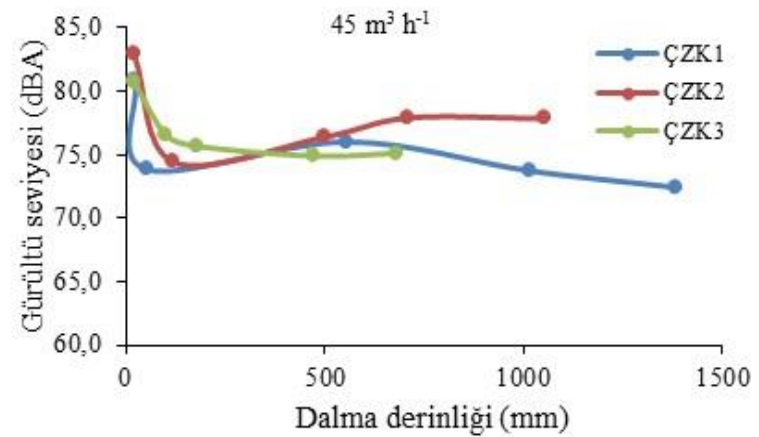

(b)

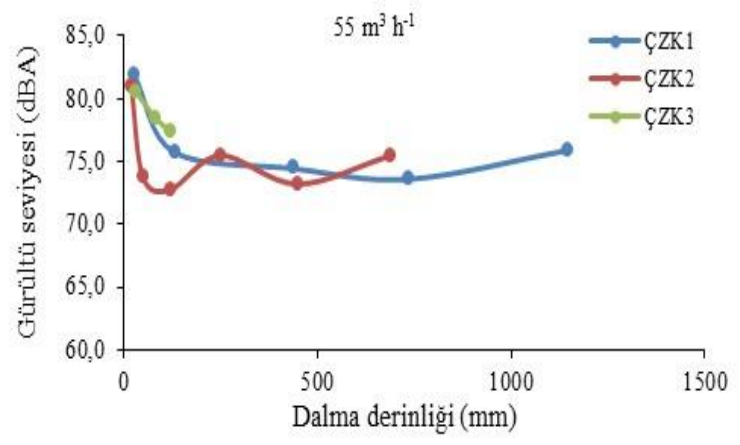

(d)

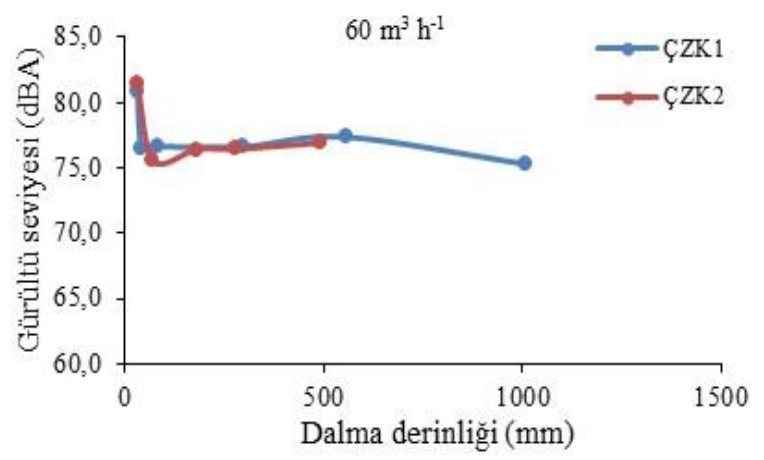

(e)

Şekil 10. Farklı çakıl zonu kalınlıklarında pompanın dalma derinliğine bağlı gürültü seviyesi değişimi.

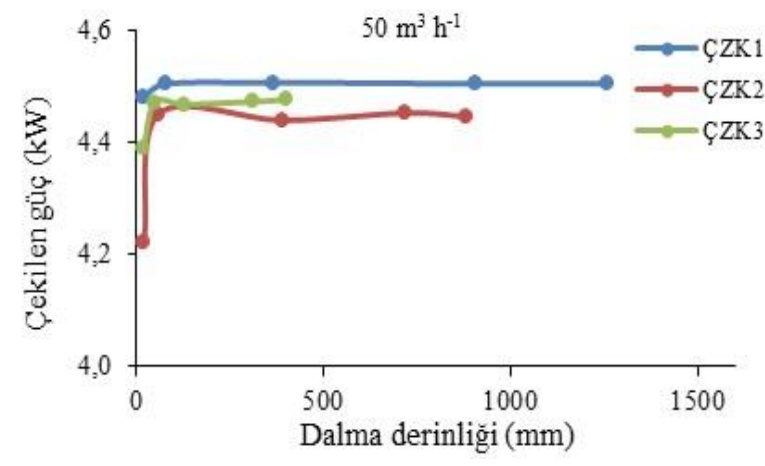

(a)

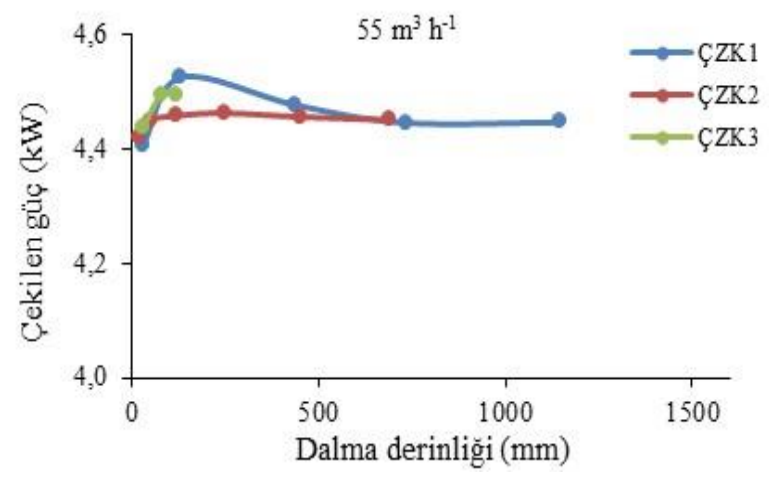

(b)

Şekil 11. Farklı çakıl zonu kalınlığında pompanın 50 ve $55 \mathrm{~m}^{3} \mathrm{~h}^{-1}$ debilerinde dalma derinliği ve çekilen güç arasındaki ilişki. 
Bütün çakıl zonu kombinasyonların da dalma derinliği değişimine bağlı çekilen güçte belirgin bir değişim görülmemiştir. Ancak gürültü seviyesinde ki gibi pompaya hava girişinin olduğu anlarda güç değerlerinde ani düşüşler görülmüştür (Şekil 11).

\section{Sonuç ve Öneriler}

Çakıl zonu kalınlıklarının pompaların kritik dalma derinliklerine doğrudan bir etkisi saptanmamıştır. Ancak çakıl zonu kalınlıklarının artması düşümü artırdığından dolayı pompanın kritik dalma derinliği seviyesine ulaşmasını hızlandırmış, hatta kalın çakıl zonlarının yüksek debilerinde kritik değerin altında çalışmasına neden olmuştur. Örneğin pompanın $50 \mathrm{~m}^{3} \mathrm{~h}^{-1}$ debisinde ve tam açık vana değerinde elde edilen dalma derinliği seviyeleri, pompanın kritik dalma derinliğinin ÇZK1, ÇZK2 ve ÇZK3 'de sırası ile 3, 2.1 ve 0.92 katı çalışma aralığına sahip olduğu görülmüştür. ÇZK2'de $60 \mathrm{~m}^{3} \mathrm{~h}^{-1}$ debide, ÇZK3'de 50 ve $55 \mathrm{~m}^{3} \mathrm{~h}^{-1}$ debi değerlerinde pompanın tüm dalma derinlikleri seviyesi kritik değerin altında çalışmıştır. Bu bakımdan literatürler de belirtildiği gibi çakıl zonu kalınlığını arttırmanın kuyu verimine katkısı olmadığı gibi pompaların kritik dalma derinliğine ulaşmasını hızlandırmaktadır.

\section{Kaynaklar}

Akpınar, K. 1999. Su Sondaj Kuyularının Açılması ve İşletilmesi Sırasında Çıkan Sorunlar ve Çözümleri. ISBN 975-94033-0-7, Ankara.

Anonim, 1998. American National Standard for Pump Intake Design. Hydraulic Institu, New Jersey.

Anonim, 2002. Rotodinamik Pompalar-Hidrolik Performans Kabul Deneyleri, Sınıf 1 ve Sınıf 2. Türk Standardları Enstitüsü, Ankara.

Anonim, 2014. For pumps-submersible-clean water. Ankara, Turkish Standards Institute.

Boman, B., Shukla, S., Hardin, J. 2003. Design and Construction of Screened Wells for Agricultural Irrigation Systems. EDIS University of Florida.
Čdina, M., 2003. Detection of cavitation phenomenon in a centrifugal pump using audible sound. Mechanical systems and Signal Processing, 17: 1335-1347.

Christiansen, C. 2005. Pumping from Shallow Streams in: Mines, N.R.a. (Ed.), Natural Resource Sciences p. 2.

Çalışır, S., Eryılmaz, T., Hacıseferoğulları, H., Mengeş, H.O. 2007. Santrifüj Pompalarda Gürültü. Tarım Makinaları Bilimi Dergisi.

Çalışır, S., 2009. Sulamada Pompaj Tesisleri. Tarım Makinaları, Editör: Gazanfer Ergüneş. Nobel Yayınları, 351-413.

Çebi, T. 1994. Yeraltı Suyunda İçme ve Kullanma Suyu Temin Amaçlı Kuyularda Tasarım Teknikleri. Jeoloji Mühensiliği Dergisi, 70-87.

Hanson, B. 2000. Irrigation Pumping Plants (UC Irrigation and Drainage Specialist), Department of Land, Air and Water Resources, University of California, Davis.

Möller, G., Detert, M., Boes, R.M. 2015. Vortexinduced air entrainment rates at intakes. Journal of Hydraulic Engineering, 141, 04015026.

Okamura, T., Kamemoto, K., Matsui, J. 2007. CFD Prediction and Model Experiment on Suction Vortices in Pump Sump.

Polak, K., Kaznowska-Opala, K., Pawlecka, K. 2016. Causes of Decreased discharge and damage to a dewatering well's gravel coat. Mine Water and the Environment, 35: 120-127.

Rafferty, K., 2001. Specification of water wells. GeoHeat Center.

Sarkardeh, H. 2017. Minimum Reservoir water level in hydropower dams. Chinese Journal of Mechanical Engineering, 30: 1017-1024.

Yildirim, N., Akay, H., Taştan, K. 2011. Critical submergence for multiple pipe intakes by the potential flow solution. Journal of Hydraulic Research, 49: 117-121. 


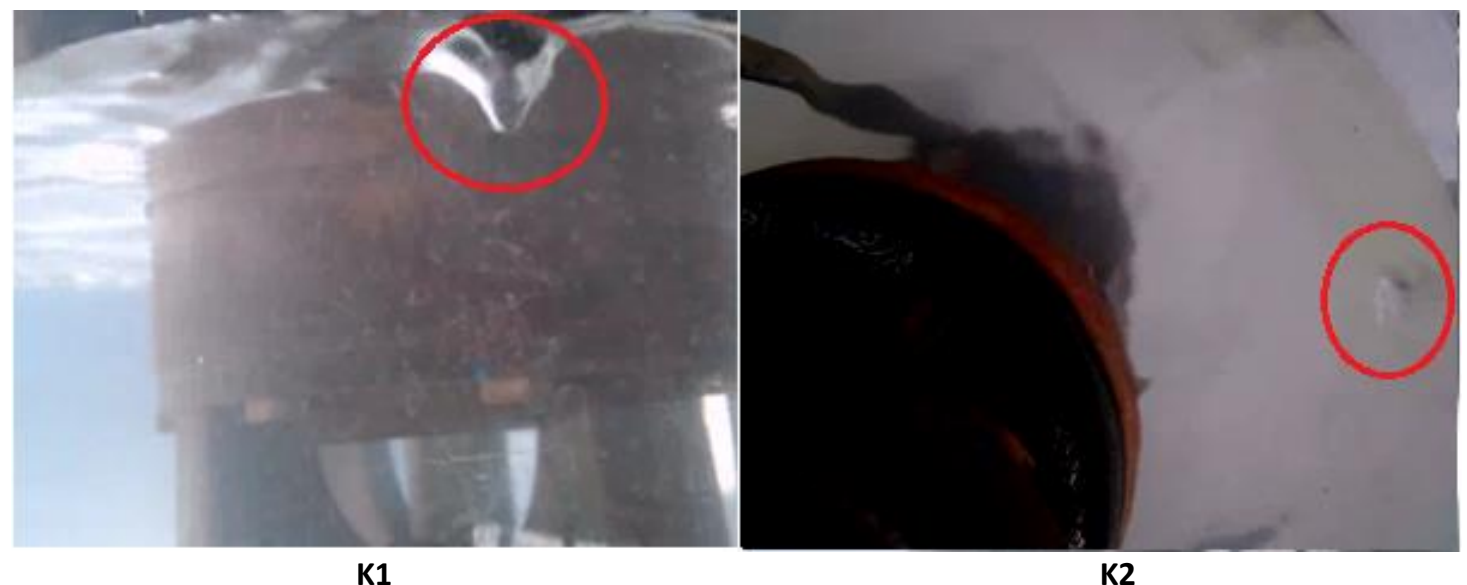

Ek Şekil 1. ÇZK1; $Q=60 \mathrm{~m}^{3} \mathrm{~h}^{-1} ; \mathrm{S}=80 \mathrm{~mm}$

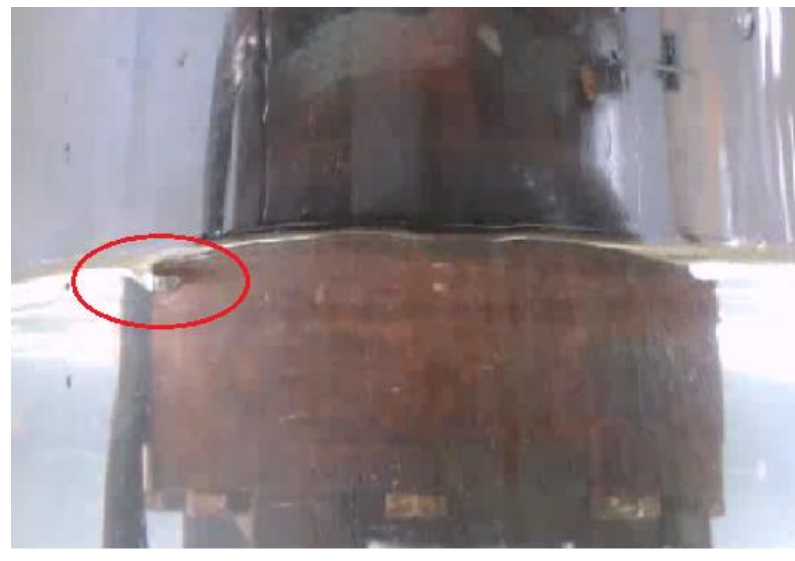

K1

Ek Şekil 2. ÇZK2; $Q=50 \mathrm{~m}^{3} \mathrm{~h}^{-1} ; \mathrm{S}=60 \mathrm{~mm}$

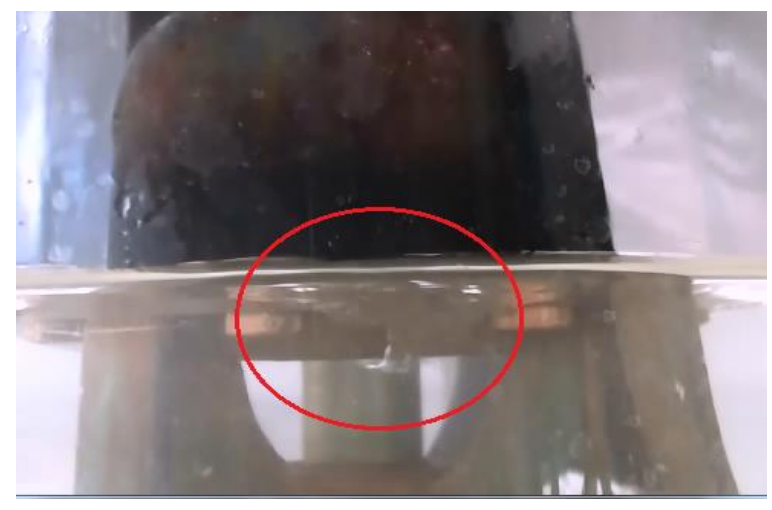

$$
\text { K1 }
$$

Ek Şekil 3. ÇZK3; $Q=45 \mathrm{~m}^{3} \mathrm{~h}^{-1} ; \mathrm{S}=20 \mathrm{~mm}$

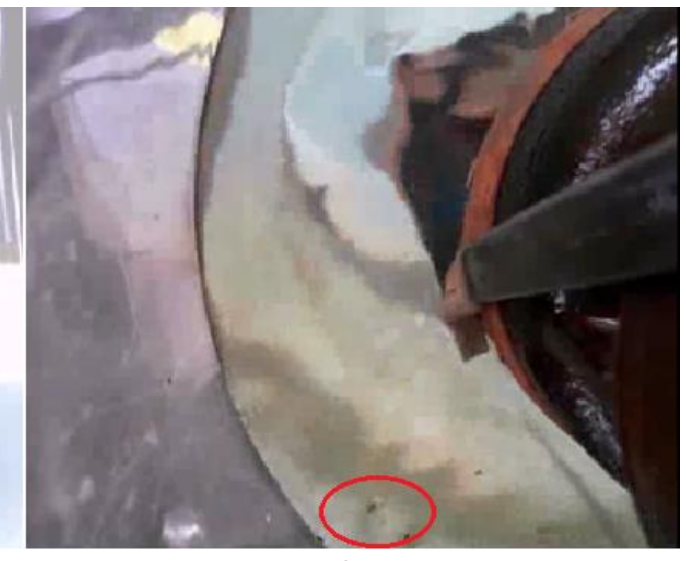

K2

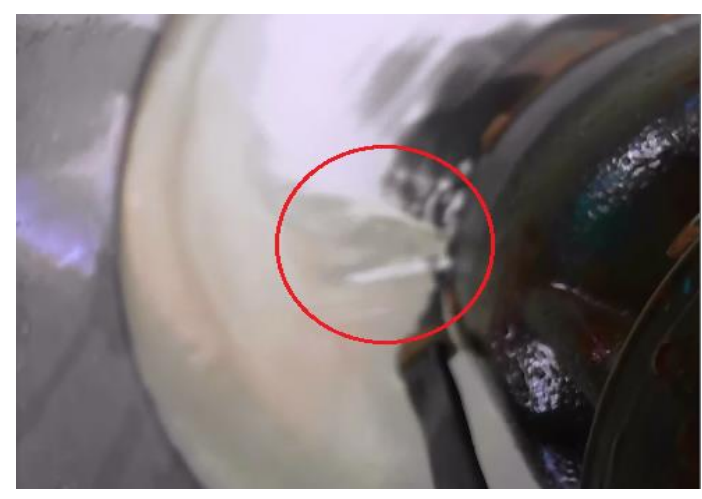

K2 
Ek Çizelge 1. ÇZK1 çakıl zonu genişliğinde elde edilen veriler

\begin{tabular}{|c|c|c|c|c|c|c|c|c|}
\hline$Q\left(m^{3} h^{-1}\right)$ & $S^{2}(\mathrm{~cm})$ & $\Delta^{2}(\mathrm{~cm})$ & G (dBA) & $\mathbf{N}(\mathbf{k W})$ & $\mathrm{V} 1\left(\mathrm{~m} \mathrm{~s}^{-1}\right)$ & $\mathrm{V} 2\left(\mathrm{~m} \mathrm{~s}^{-1}\right)$ & $\mathrm{Pb}(\mathrm{kPa})$ & $\mathrm{Pe}^{2}(\mathrm{kPa})$ \\
\hline${ }^{1} 40.1 \pm 0.007$ & $147 \pm 0.57$ & $41 \pm 0.57$ & $73.5 \pm 0.13$ & $4.3 \pm 0.0016$ & \multirow{5}{*}{$0.23 \pm 0.001$} & $2.31 \pm 0.001$ & $146.5 \pm 0.1$ & $10.47 \pm 0.057$ \\
\hline $40.1 \pm 0.006$ & $102.3 \pm 0.88$ & $85.6 \pm 0.88$ & $74.9 \pm 0.19$ & $4.30 \pm 0.0014$ & & $2.32 \pm 0.001$ & $141.5 \pm 0.1$ & $14.87 \pm 0.088$ \\
\hline $40.1 \pm 0.002$ & $60.1 \pm 0.72$ & $127.8 \pm 0.72$ & $74.3 \pm 0.122$ & $4.30 \pm 0.0014$ & & $2.32 \pm 0.001$ & $137.4 \pm 0.1$ & $19.01 \pm 0.072$ \\
\hline $40.2 \pm 0.007$ & $5 \pm 0.57$ & $183 \pm 0.57$ & $76.5 \pm 0.15$ & $4.31 \pm 0.0013$ & & $2.32 \pm 0.001$ & $131.6 \pm 0.1$ & $24.42 \pm 0.057$ \\
\hline $36.8 \pm 0.05$ & $2.1 \pm 0.16$ & $185.8 \pm 0.16$ & $81.3 \pm 0.1$ & $3.96 \pm 0.0041$ & & $2.13 \pm 0.003$ & $110.6 \pm 0.6$ & $24.29 \pm 0.016$ \\
\hline $145.0 \pm 0.01$ & $138.3 \pm 0.88$ & $49.6 \pm 0.88$ & $72.4 \pm 0.12$ & $4.38 \pm 0.0013$ & \multirow{5}{*}{$0.26 \pm 0.001$} & $2.60 \pm 0.001$ & $132.4 \pm 0.1$ & $12.02 \pm 0.092$ \\
\hline $45.1 \pm 0.007$ & $101.3 \pm 0.88$ & $86.6 \pm 0.88$ & $73.7 \pm 0.098$ & $4.38 \pm 0.0011$ & & $2.61 \pm 0.001$ & $129.6 \pm 0.01$ & $15.68 \pm 0.092$ \\
\hline $44.9 \pm 0.009$ & $55.5 \pm 0.86$ & $132.5 \pm 0.86$ & $76.0 \pm 0.16$ & $4.4 \pm 0.0009$ & & $2.60 \pm 0.001$ & $124.3 \pm 0.1$ & $20.15 \pm 0.091$ \\
\hline $45.0 \pm 0.007$ & $5.1 \pm 0.44$ & $182.8 \pm 0.44$ & $73.8 \pm 0.22$ & $4.4 \pm 0.0015$ & & $2.61 \pm 0.001$ & $118.9 \pm 0.1$ & $25.12 \pm 0.046$ \\
\hline $43.7 \pm 0.035$ & $2.2 \pm 0.14$ & $185.7 \pm 0.14$ & $80.9 \pm 0.14$ & $4.3 \pm 0.0022$ & & $2.53 \pm 0.002$ & $115.0 \pm 0.2$ & $25.2 \pm 0.015$ \\
\hline $150.1 \pm 0.008$ & $125.8 \pm 0.6$ & $62.1 \pm 0.6$ & $68.1 \pm 0.11$ & $4.5 \pm 0.0008$ & \multirow{5}{*}{$0.29 \pm 0.001$} & $2.90 \pm 0.001$ & $116.7 \pm 0.1$ & $14.07 \pm 0.064$ \\
\hline $50.2 \pm 0.007$ & $90.6 \pm 0.4$ & $97.4 \pm 0.4$ & $73.0 \pm 0.18$ & $4.5 \pm 0.0007$ & & $2.90 \pm 0.001$ & $113.6 \pm 0.2$ & $17.52 \pm 0.043$ \\
\hline $50.1 \pm 0.007$ & $36.5 \pm 0.95$ & $151.5 \pm 0.95$ & $73.8 \pm 0.11$ & $4.5 \pm 0.0006$ & & $2.90 \pm 0.001$ & $108.3 \pm 0.2$ & $22.83 \pm 0.1$ \\
\hline $49.9 \pm 0.007$ & $7.9 \pm 0.21$ & $180.1 \pm 0.21$ & $76.1 \pm 0.12$ & $4.5 \pm 0.0007$ & & $2.89 \pm 0.001$ & $105.6 \pm 0.2$ & $25.6 \pm 0.023$ \\
\hline $48.9 \pm 0.013$ & $2.1 \pm 0.12$ & $185.8 \pm 0.12$ & $78.9 \pm 0.18$ & $4.4 \pm 0.0017$ & & $2.83 \pm 0.001$ & $105.0 \pm 0.2$ & $26 \pm 0.012$ \\
\hline${ }^{1} 55.1 \pm 0.01$ & $114.5 \pm 0.76$ & $73.5 \pm 0.76$ & $75.9 \pm 0.21$ & $4.4 \pm 0.0016$ & \multirow{5}{*}{$0.31 \pm 0.001$} & $3.18 \pm 0.001$ & $100.3 \pm 0.2$ & $16.02 \pm 0.074$ \\
\hline $54.7 \pm 0.07$ & $73.5 \pm 0.23$ & $114.5 \pm 0.23$ & $73.6 \pm 0.15$ & $4.4 \pm 0.0015$ & & $3.16 \pm 0.004$ & $99.0 \pm 0.3$ & $19.98 \pm 0.022$ \\
\hline $55.1 \pm 0.05$ & $43.9 \pm 0.15$ & $144.1 \pm 0.15$ & $74.5 \pm 0.18$ & $4.4 \pm 0.0037$ & & $3.19 \pm 0.003$ & $94.1 \pm 0.3$ & $22.98 \pm 0.014$ \\
\hline $54.7 \pm 0.06$ & $13.1 \pm 0.37$ & $174.9 \pm 0.37$ & $75.8 \pm 0.19$ & $4.5 \pm 0.0013$ & & $3.16 \pm 0.004$ & $91.8 \pm 0.3$ & $25.91 \pm 0.036$ \\
\hline $53.2 \pm 0.02$ & $2.8 \pm 0.33$ & $185.2 \pm 0.33$ & $81.9 \pm 0.12$ & $4.4 \pm 0.0026$ & & $3.08 \pm 0.001$ & $83.6 \pm 0.3$ & $26.67 \pm 0.036$ \\
\hline${ }^{1} 60.1 \pm 0.019$ & $100.9 \pm 0.58$ & $87.1 \pm 0.58$ & $75.3 \pm 0.29$ & $4.4 \pm 0.0007$ & \multirow{6}{*}{$0.34 \pm 0.001$} & $3.47 \pm 0.001$ & $76.5 \pm 0.2$ & $18.31 \pm 0.056$ \\
\hline $59.9 \pm 0.01$ & $55.5 \pm 0.35$ & $132.5 \pm 0.35$ & $77.3 \pm 0.15$ & $4.4 \pm 0.0007$ & & $3.47 \pm 0.001$ & $75.5 \pm 0.2$ & $22.76 \pm 0.034$ \\
\hline $60.2 \pm 0.01$ & $29.7 \pm 0.17$ & $158.3 \pm 0.17$ & $76.6 \pm 0.18$ & $4.4 \pm 0.0004$ & & $3.48 \pm 0.001$ & $71.3 \pm 0.2$ & $25.33 \pm 0.017$ \\
\hline $59.9 \pm 0.01$ & $8.1 \pm 0.4$ & $179.9 \pm 0.4$ & $76.6 \pm 0.17$ & $4.4 \pm 0.0006$ & & $3.47 \pm 0.001$ & $69.9 \pm 0.1$ & $27.41 \pm 0.039$ \\
\hline $60.0 \pm 0.009$ & $4 \pm 0.26$ & $184 \pm 0.26$ & $76.5 \pm 0.16$ & $4.4 \pm 0.0012$ & & $3.47 \pm 0.001$ & $68.7 \pm 0.1$ & $27.82 \pm 0.025$ \\
\hline $58.9 \pm 0.015$ & $3.1 \pm 0.18$ & $184.9 \pm 0.18$ & $80.9 \pm 0.22$ & $4.3 \pm 0.0028$ & & $3.41 \pm 0.001$ & $67.8 \pm 0.2$ & $27.71 \pm 0.018$ \\
\hline
\end{tabular}

\footnotetext{
: Bu satırlardaki veriler, kuyu besleme borularının her ikisinin tam açık olduğu durumda alınmıştır.
}

${ }^{2}$ :Bu sütunlardaki veriler 5 adet diğer sütunlar 50 adet verinin ortalamasından oluşmaktadır. Deney koşulları: FT1; ÇZK1; FU1

$\mathrm{S}_{\text {mak }}: 188 \mathrm{~cm} ; \mathrm{H}_{\mathrm{s}}: 89 \mathrm{~cm} \mathrm{~T}_{\text {hava }}\left({ }^{\circ} \mathrm{C}\right): 14,4 \pm 0,5 ; \mathrm{T}_{\mathrm{su}}\left({ }^{0} \mathrm{C}\right): 9,5 \pm 0,22 ; \mathrm{BN}: \% 47 \pm 1,4$

Deney tarihi: 03-04/11/2015; Net deney süresi: 205 dakika 
Ek Çizelge 2. ÇZK2 çakıl zonu genişliğinde elde edilen veriler

\begin{tabular}{|c|c|c|c|c|c|c|c|c|}
\hline$Q\left(m^{3} h^{-1}\right)$ & $\mathrm{S}^{2}(\mathrm{~cm})$ & $\Delta^{2}(\mathrm{~cm})$ & G (dBA) & $\mathbf{N}(\mathbf{k W})$ & $\mathrm{V} 1\left(\mathrm{~m} \mathrm{~s}^{-1}\right)$ & $\mathrm{V} 2\left(\mathrm{~m} \mathrm{~s}^{-1}\right)$ & $\mathrm{Pb}(\mathrm{kPa})$ & $\mathrm{Pe}^{2}(\mathrm{kPa})$ \\
\hline${ }^{1} 40.2 \pm 0.006$ & $122.33 \pm 0.60$ & $65.66 \pm 0.60$ & $72.5 \pm 0.15$ & $4.4 \pm 0.0009$ & \multirow{5}{*}{$0.23 \pm 0.001$} & $2.32 \pm 0.001$ & $144.8 \pm 0.1$ & $12.89 \pm 0.06$ \\
\hline $40.1 \pm 0.006$ & $71.36 \pm 0.44$ & $116.63 \pm 0.44$ & $79.1 \pm 0.21$ & $4.41 \pm 0.00079$ & & $2.32 \pm 0.001$ & $140.8 \pm 0.1$ & $17.94 \pm 0.044$ \\
\hline $40.1 \pm 0.005$ & $29.16 \pm 0.6$ & $158.83 \pm 0.6$ & $75.4 \pm 0.14$ & $4.4 \pm 0.0007$ & & $2.32 \pm 0.001$ & $135.7 \pm 0.1$ & $12.06 \pm 0.06$ \\
\hline $40.1 \pm 0.008$ & $5 \pm 0.57$ & $183 \pm 0.57$ & $74.2 \pm 0.1$ & $4.4 \pm 0.001$ & & $2.32 \pm 0.001$ & $133.4 \pm 0.1$ & $24.42 \pm 0.057$ \\
\hline $37.1 \pm 0.03$ & $2.16 \pm 0.16$ & $185.83 \pm 0.16$ & $82.8 \pm 0.09$ & $4.1 \pm 0.006$ & & $2.14 \pm 0.001$ & $116.1 \pm 0.9$ & $24.32 \pm 0.016$ \\
\hline $145.1 \pm 0.008$ & $105.43 \pm 0.74$ & $82.56 \pm 0.74$ & $77.8 \pm 0.2$ & $4.4 \pm 0.0015$ & \multirow{5}{*}{$0.26 \pm 0.001$} & $2.61 \pm 0.001$ & $130.3 \pm 0.1$ & $15.32 \pm 0.078$ \\
\hline $45.0 \pm 0.006$ & $70.8 \pm 0.34$ & $117.2 \pm 0.34$ & $77.9 \pm 0.18$ & $4.4 \pm 0.0018$ & & $2.60 \pm 0.001$ & $128.3 \pm 0.1$ & $18.63 \pm 0.036$ \\
\hline $45.1 \pm 0.007$ & $49.86 \pm 0.23$ & $138.13 \pm 0.23$ & $76.4 \pm 0.14$ & $4.4 \pm 0.0014$ & & $2.61 \pm 0.001$ & $124.3 \pm 0.1$ & $20.71 \pm 0.024$ \\
\hline $45.1 \pm 0.007$ & $12.06 \pm 0.14$ & $175.93 \pm 0.14$ & $74.4 \pm 0.15$ & $4.4 \pm 0.0017$ & & $2.60 \pm 0.001$ & $120.7 \pm 0.1$ & $24.41 \pm 0.015$ \\
\hline $40.6 \pm 0.02$ & $2.23 \pm 0.18$ & $185.76 \pm 0.18$ & $82.9 \pm 0.11$ & $4.1 \pm 0.007$ & & $2.35 \pm 0.001$ & $100.7 \pm 1$ & $24.78 \pm 0.019$ \\
\hline${ }^{1} 50.1 \pm 0.006$ & $87.96 \pm 0.82$ & $100.03 \pm 0.82$ & $73.7 \pm 0.14$ & $4.4 \pm 0.0015$ & \multirow{5}{*}{$0.29 \pm 0.001$} & $2.90 \pm 0.001$ & $113.18 \pm 0.1$ & $17.79 \pm 0.088$ \\
\hline $50.1 \pm 0.008$ & $72 \pm 0.26$ & $116 \pm 0.26$ & $77.1 \pm 0.2$ & $4.4 \pm 0.0014$ & & $2.89 \pm 0.001$ & $111.3 \pm 0.1$ & $19.34 \pm 0.028$ \\
\hline $50.1 \pm 0.009$ & $39 \pm 0.23$ & $149 \pm 0.23$ & $73.8 \pm 0.16$ & $4.4 \pm 0.0014$ & & $2.90 \pm 0.001$ & $107.8 \pm 0.1$ & $22.59 \pm 0.024$ \\
\hline $50.0 \pm 0.007$ & $5.86 \pm 0.12$ & $182.13 \pm 0.12$ & $74.2 \pm 0.14$ & $4.4 \pm 0.0015$ & & $2.89 \pm 0.001$ & $105.6 \pm 0.1$ & $25.81 \pm 0.012$ \\
\hline $45.9 \pm 0.02$ & $2.2 \pm 0.11$ & $185.8 \pm 0.11$ & $83.4 \pm 0.07$ & $4.2 \pm 0.003$ & & $2.65 \pm 0.001$ & $93.1 \pm 0.4$ & $25.55 \pm 0.012$ \\
\hline${ }^{1} 54.9 \pm 0.011$ & $68.86 \pm 0.4$ & $119.13 \pm 0.4$ & $75.4 \pm 0.2$ & $4.4 \pm 0.0016$ & \multirow{6}{*}{$0.31 \pm 0.001$} & $3.17 \pm 0.001$ & $96.1 \pm 0.2$ & $20.48 \pm 0.039$ \\
\hline $55.1 \pm 0.008$ & $44.8 \pm 0.15$ & $143.2 \pm 0.15$ & $73.2 \pm 0.13$ & $4.4 \pm 0.0016$ & & $3.18 \pm 0.001$ & $92.8 \pm 0.1$ & $22.87 \pm 0.014$ \\
\hline $55.1 \pm 0.007$ & $24.86 \pm 0.39$ & $163.13 \pm 0.39$ & $75.4 \pm 0.15$ & $4.4 \pm 0.0016$ & & $3.18 \pm 0.001$ & $91.3 \pm 0.1$ & $24.82 \pm 0.038$ \\
\hline $55.0 \pm 0.0122$ & $11.83 \pm 0.17$ & $176.16 \pm 0.17$ & $72.7 \pm 0.12$ & $4.4 \pm 0.0012$ & & $3.18 \pm 0.001$ & $90.3 \pm 0.2$ & $26.08 \pm 0.017$ \\
\hline $54.9 \pm 0.007$ & $5.03 \pm 0.12$ & $182.96 \pm 0.12$ & $73.7 \pm 0.19$ & $4.4 \pm 0.0017$ & & $3.12 \pm 0.001$ & $89.8 \pm 0.1$ & $26.58 \pm 0.011$ \\
\hline $53.9 \pm 0.013$ & $2.03 \pm 0.13$ & $185.96 \pm 0.13$ & $81.0 \pm 0.2$ & $4.4 \pm 0.002$ & & $3.12 \pm 0.001$ & $88.1 \pm 0.9$ & $26.87 \pm 0.013$ \\
\hline${ }^{1} 60.0 \pm 0.009$ & $49.17 \pm 0.28$ & $138.83 \pm 0.28$ & $76.9 \pm 0.15$ & $4.4 \pm 0.0016$ & \multirow{5}{*}{$0.34 \pm 0.001$} & $3.47 \pm 0.001$ & $74.3 \pm 0.1$ & $23.42 \pm 0.028$ \\
\hline $60.1 \pm 0.008$ & $27.99 \pm 0.23$ & $160.00 \pm 0.23$ & $76.4 \pm 0.14$ & $4.4 \pm 0.0014$ & & $3.47 \pm 0.001$ & $72.4 \pm 0.2$ & $25.49 \pm 0.022$ \\
\hline $60.1 \pm 0.008$ & $18.08 \pm 0.1$ & $169.91 \pm 0.1$ & $76.4 \pm 0.1$ & $4.4 \pm 0.0014$ & & $3.47 \pm 0.001$ & $71.7 \pm 0.2$ & $26.47 \pm 0.01$ \\
\hline $59.9 \pm 0.007$ & $7.11 \pm 0.26$ & $180.88 \pm 0.26$ & $75.5 \pm 0.14$ & $4.4 \pm 0.0014$ & & $3.47 \pm 0.001$ & $70.8 \pm 0.2$ & $27.53 \pm 0.026$ \\
\hline $58.9 \pm 0.012$ & $2.90 \pm 0.27$ & $185.09 \pm 0.27$ & $81.4 \pm 0.2$ & $4.3 \pm 0.003$ & & $3.41 \pm 0.001$ & $69.8 \pm 0.3$ & $27.72 \pm 0.026$ \\
\hline
\end{tabular}

${ }^{1}$ : Bu satırlardaki veriler, kuyu besleme borularının her ikisinin tam açık olduğu durumda alınmıştır.

2: Bu sütunlardaki veriler 5 adet diğer sütunlar 50 adet verinin ortalamasından oluşmaktadır.

Deney koşulları: FT1; ÇZK2; FU1

$S_{\text {mak: }} 188 \mathrm{~cm} ; \mathrm{H}_{\mathrm{s}}: 89 \mathrm{~cm} \mathrm{~T}_{\text {hava }}\left({ }^{0} \mathrm{C}\right): 19,53 \pm 0,07 ; \mathrm{T}_{\mathrm{su}}\left({ }^{0} \mathrm{C}\right): 15,32 \pm 0,062 ; \mathrm{BN}: \% 47 \pm 0,13$

Deney tarihi: 08/10/2015; Net deney süresi: 165 dakika 
Ek Çizelge 3. Alttan beslemede ÇZK3 çakıl zonu genişliğinde elde edilen veriler

\begin{tabular}{|c|c|c|c|c|c|c|c|c|}
\hline$Q\left(m^{3} h^{-1}\right)$ & $\mathrm{S}^{2}(\mathrm{~cm})$ & $\Delta^{2}(\mathrm{~cm})$ & G (dBA) & $\mathbf{N}(\mathrm{kW})$ & $\mathrm{V} 1\left(\mathrm{~m} \mathrm{~s}^{-1}\right)$ & $\mathrm{V} 2\left(\mathrm{~m} \mathrm{~s}^{-1}\right)$ & $\mathrm{Pb}(\mathrm{kPa})$ & $\mathrm{Pe}^{2}(\mathrm{kPa})$ \\
\hline $139.9 \pm 0.008$ & $93.93 \pm 0.21$ & $94.06 \pm 0.21$ & $77.7 \pm 0.22$ & $4.3 \pm 0.002$ & & $2.31 \pm 0.001$ & $140.8 \pm 0.1$ & $15.74 \pm 0.021$ \\
\hline $40.1 \pm 0.012$ & $56.06 \pm 0.37$ & $131.93 \pm 0.37$ & $78.1 \pm 0.15$ & $4.3 \pm 0.0018$ & & $2.32 \pm 0.001$ & $136.2 \pm 0.2$ & $19.41 \pm 0.037$ \\
\hline $40.1 \pm 0.04$ & $29.76 \pm 0.14$ & $158.23 \pm 0.14$ & $77.2 \pm 0.11$ & $4.3 \pm 0.001$ & $0.23 \pm 0.001$ & $2.32 \pm 0.001$ & $134.8 \pm 0.1$ & $21.97 \pm 0.014$ \\
\hline $40.0 \pm 0.01$ & $13.16 \pm 0.24$ & $174.83 \pm 0.24$ & $74.0 \pm 0.19$ & $4.3 \pm 0.0015$ & & $2.31 \pm 0.001$ & $132.8 \pm 0.2$ & $23.63 \pm 0.024$ \\
\hline $37.7 \pm 0.04$ & $2.04 \pm 0.24$ & $185.95 \pm 0.24$ & $82.2 \pm 0.09$ & $4.2 \pm 0.0019$ & & $2.18 \pm 0.001$ & $124.9 \pm 0.4$ & $24.31 \pm 0.024$ \\
\hline $144.9 \pm 0.009$ & $68.03 \pm 0.14$ & $119.96 \pm 0.14$ & $75.1 \pm 0.17$ & $4.4 \pm 0.0018$ & & $2.59 \pm 0.001$ & $125.1 \pm 0.2$ & $18.95 \pm 0.015$ \\
\hline $45.0 \pm 0.009$ & $46.94 \pm 0.22$ & $141.05 \pm 0.22$ & $74.9 \pm 0.17$ & $4.4 \pm 0.0019$ & & $2.60 \pm 0.001$ & $122.6 \pm 0.2$ & $20.98 \pm 0.023$ \\
\hline $45.0 \pm 0.011$ & $17.99 \pm 0.21$ & $170.01 \pm 021$ & $75.6 \pm 0.22$ & $4.4 \pm 0.0018$ & $0.26 \pm 0.001$ & $2.60 \pm 0.001$ & $119.2 \pm 0.2$ & $23.85 \pm 0.022$ \\
\hline $44.9 \pm 0.009$ & $9.96 \pm 0.21$ & $178.03 \pm 0.21$ & $76.5 \pm 0.16$ & $4.4 \pm 0.002$ & & $2.60 \pm 0.001$ & $118.8 \pm 0.1$ & $24.61 \pm 0.022$ \\
\hline $43.3 \pm 0.03$ & $2.01 \pm 0.31$ & $185.98 \pm 0.31$ & $80.7 \pm 0.13$ & $4.3 \pm 0.002$ & & $2.50 \pm 0.001$ & $116.2 \pm 0.3$ & $24.78 \pm 0.033$ \\
\hline${ }^{1} 50.1 \pm 0.011$ & $39.98 \pm 0.26$ & $148.02 \pm 0.26$ & $76.4 \pm 0.15$ & $4.4 \pm 0.001$ & & $2.90 \pm 0.001$ & $106.7 \pm 0.2$ & $22.5 \pm 0.028$ \\
\hline $49.9 \pm 0.011$ & $30.93 \pm 0.22$ & $157.06 \pm 0.22$ & $78.1 \pm 0.29$ & $4.4 \pm 0.0013$ & & $2.89 \pm 0.001$ & $106.5 \pm 0.1$ & $23.36 \pm 0.023$ \\
\hline $49.9 \pm 0.011$ & $13.03 \pm 0.26$ & $174.96 \pm 0.26$ & $76.6 \pm 0.20$ & $4.4 \pm 0.0018$ & $0.29 \pm 0.001$ & $2.89 \pm 0.001$ & $103.7 \pm 0.2$ & $25.14 \pm 0.028$ \\
\hline $50.0 \pm 0.02$ & $5.01 \pm 0.12$ & $182.98 \pm 0.12$ & $77.7 \pm 0.21$ & $4.4 \pm 0.0084$ & & $2.89 \pm 0.001$ & $103.9 \pm 0.2$ & $25.91 \pm 0.012$ \\
\hline $48.3 \pm 0.035$ & $2.15 \pm 0.35$ & $185.85 \pm 0.35$ & $80.8 \pm 0.14$ & $4.3 \pm 0.0033$ & & $2.79 \pm 0.002$ & $100.2 \pm 0.2$ & $25.55 \pm 0.037$ \\
\hline${ }^{1} 54.9 \pm 0.015$ & $11.97 \pm 0.26$ & $176.03 \pm 0.26$ & $77.4 \pm 0.18$ & $4.4 \pm 0.0015$ & & $3.18 \pm 0.001$ & $90.4 \pm 0.2$ & $26.07 \pm 0.025$ \\
\hline $54.9 \pm 0.043$ & $7.91 \pm 0.14$ & $180.08 \pm 0.14$ & $78.4 \pm 0.27$ & $4.4 \pm 0.0017$ & $0.31 \pm 0.001$ & $3.17 \pm 0.003$ & $90.5 \pm 0.4$ & $26.5 \pm 0.014$ \\
\hline $55.0 \pm 0.016$ & $3 \pm 0.1$ & $185 \pm 0.1$ & $80.5 \pm 0.15$ & $4.4 \pm 0.0031$ & & $3.18 \pm 0.001$ & $83.3 \pm 0.3$ & $26.98 \pm 0.010$ \\
\hline
\end{tabular}

${ }^{1}$ : Bu satırlardaki veriler, kuyu besleme borularının her ikisinin tam açık olduğu durumda alınmıştır.

2: Bu sütunlardaki veriler 5 adet diğer sütunlar 50 adet verinin ortalamasından oluşmaktadır.

Deney koşulları: FT1; ÇZK3; FU1

$S_{\text {mak: }} 188 \mathrm{~cm} ; \mathrm{H}_{\mathrm{s}}: 89 \mathrm{~cm} \mathrm{~T}_{\text {hava }}\left({ }^{0} \mathrm{C}\right): 10 \pm 0,04 ; \mathrm{T}_{\text {su }}\left({ }^{0} \mathrm{C}\right): 9 \pm 0,04 ; \mathrm{BN}: \% 39,7 \pm 0,5$

Deney tarihi: 10/11/2015; Net deney süresi: 155 dakika 